\title{
OPTIMALITY CONDITIONS FOR NONCONVEX MULTISTATE CONTROL PROBLEMS IN THE COEFFICIENTS*
}

\author{
JUAN CASADO-DÍAZ ${ }^{\dagger}$, JULIO COUCE-CALVO ${ }^{\dagger}$, AND JOSÉ D. MARTÍN-GÓMEZ ${ }^{\dagger}$
}

\begin{abstract}
The purpose of this paper is to attain some optimality conditions for the identification of a diffusion matrix (material) under several restrictions. Assuming that the set of such diffusion matrices is closed for the $H$-convergence, we give a method to obtain admissible directions which applies to a not-necessarily convex control set. Our results permit obtaining the diffusion matrix from the state functions.
\end{abstract}

Key words. control in the coefficients, admissible directions, nonconvex control set

AMS subject classification. $49 \mathrm{~K} 20$

DOI. $10.1137 /$ S0363012902411714

1. Introduction. The problem we consider in the present paper is related to the choice of an optimal material under several conditions, or the identification of a material from a finite number of observations. In a mathematical setting, we have the model problem

$$
\min _{A \in M(\Omega)} J\left(y_{1}, \ldots, y_{k}\right)
$$

where $y_{i}=y_{i}(A), 1 \leq i \leq k$, are the solutions of the equations

$$
\begin{cases}-\operatorname{div} A \nabla y_{i}=f_{i} & \text { in } \mathcal{D}^{\prime}(\Omega) \\ y_{i} \in H_{0}^{1}(\Omega), & 1 \leq i \leq k\end{cases}
$$

Here $\Omega$ is a bounded open set of $\mathbf{R}^{N}, J$ is a smooth objective functional in $H_{0}^{1}(\Omega)^{k}$, $f_{1}, \ldots, f_{k}$ are $k$ fixed elements of $H^{-1}(\Omega)$, and $M(\Omega)$ is a given set of measurable functions with values in the space of symmetric matrices of order $N$. The elements of $M(\Omega)$ are uniformly elliptic and bounded. Clearly, other generalizations can be considered: $J$ depending on $A$, other boundary conditions, etc. A physical example is the identification of a material. For this purpose, we apply a finite number $k$ of external conditions (in our case they are represented by $f_{i}$ ) and in each case we realize a measure of the corresponding state. For example, we give the value $z_{i}$ of the state in a subset $\omega \subset \Omega$. Then the problem can be formulated as

$$
\min \sum_{i=1}^{k} \int_{\omega}\left|y_{i}-z_{i}\right|^{2} d x,
$$

where $y_{i}$ are the solutions of (1.2).

Assuming $J$ is sequential lower semicontinuous for the weak topology of $H_{0}^{1}(\Omega)^{k}$ and $M(\Omega)$ is closed for the $H$-convergence or the $G$-convergence, because we are

\footnotetext{
${ }^{*}$ Received by the editors July 22, 2002; accepted for publication (in revised form) November 24, 2003; published electronically June 15, 2004. This work has been partially supported by the projects BFM 2002-00672 of the D.G.I. of the "Ministerio de Ciencia y Tecnología" of Spain and FQM309 of the "Junta de Andalucía."

http://www.siam.org/journals/sicon/43-1/41171.html

${ }^{\dagger}$ Depto. de Ecuaciones Diferenciales y Análisis Numérico, Fac. de Matemáticas, Universidad de Sevilla, C. Tarfia s/n, 41012 Sevilla, Spain (jcasadod@us.es, jcouce@us.es, jdmartin@us.es).
} 
working with symmetric matrices and thus the two concepts are equivalent (see, e.g., [22], [19], [18], [4], [25]), it is well known that (1.1) has, at least, a solution (see, e.g., [25], [17]). If $M(\Omega)$ does not satisfy this last condition and $J$ is sequential continuous for the weak topology of $H_{0}^{1}(\Omega)^{k}$, we can obtain a relaxed problem replacing $M(\Omega)$ by its $H$-closure. Thus, it is natural to assume $M(\Omega)$ is $H$-closed. The calculus of the $H$-closure of a set is a very difficult problem and there are a lot of works in this field (see, e.g., [23], [14], [13], [8], [25], [3], [16] and the references in them). In this paper we are interested in obtaining some necessary conditions which must satisfy the optimal solution of (1.1). For $k=1$, the problem has been studied in [13], [20], [8], [25], [3]. For $k>1$, there are few results to our knowledge (see [25], [6], [7], [3]).

The paper is organized as follows:

In section 3 we give a definition of admissible direction (see Definition 3.1). Then we prove that if $A$ is a solution of $(1.1), y_{1}, \ldots, y_{k}$ the corresponding state functions, $p_{1}, \ldots, p_{k}$ the solutions of (3.2) (the adjoint states), and $C$ the matrix defined by (3.3), we have

$$
\int_{\Omega} H: C d x \leq 0
$$

for every admissible direction $H$. Related results can be found, for example, in [8], [13], [25], [3]. However, in these papers, the admissible directions are of the form $H=B-A$, with $B$ in $M(\Omega)$, which needs some convexity assumptions. When $k \leq N-1$ (in particular $k=1$ ) and $M(\Omega)$ is obtained by homogenization, mixing a finite number of matrices with fixed proportions, a result of Tartar (see [25]) shows that although $M(\Omega)$ is not convex, for every $\xi_{1}, \ldots, \xi_{k} \in \mathbf{R}^{N}$, the set

$$
\left\{\left(B \xi_{1}, \ldots, B \xi_{k}\right) / B \in M(\Omega)\right\} \subset L^{\infty}(\Omega)^{k}
$$

is convex, and thus the directions $H=B-A$ can still be considered. However, this is not true for $k \geq N$ (or, in principle, for other choices of $M(\Omega)$ even if $k \leq N-1$ ). This is the reason we have given a more general definition of admissible direction.

In section 4, assuming $M(\Omega)$ local (see Definition 4.1) and closed for the $H$ convergence, we give an original method to find admissible directions following our definition. As a consequence, we obtain the main result of the paper, Theorem 4.5, where we prove that for every $A, B \in M(\Omega), l \in\{1, \ldots, N\}, W \subset \mathbf{R}^{N}$ linear subspace of dimension $l$, and every bounded measurable set $T$ of $W$, with $l$-dimensional positive measure, the matrix $H$ defined by

$$
H(x) e_{i}=(B(x)-A(x))\left(e_{i}+\frac{1}{|T|_{\ell}} \int_{T} \nabla_{z}^{W} \hat{w}_{i}(x, z) d_{\ell}(z)\right)
$$

is an admissible direction in $A$. Here $e_{1}, \ldots, e_{N}$ is the standard basis of $\mathbf{R}^{N}, \nabla_{z}^{W}$ denotes the gradient with respect to $W$, and $\hat{w}_{i}$ is the solution of the partial differential problem given by (4.4). This direction has the difficulty that $\hat{w}_{i}$ (and then $H$ ) cannot be explicitly obtained. However, we think that it can be interesting, for example, to apply a descent method in order to solve numerically problem (1.1), where we can obtain $\hat{w}_{i}$ numerically. Related to this point, an interesting question, one that we want to study in the future, is the optimal choice of $W$ and $T$ to obtain the steepest descent direction. A criterion to determine this direction (see Remark 4.9) can be to calculate the maximum of $H: C$ on the set of matrices $H$ obtained by (1.5).

Although, as we have said above, it is not possible in general to obtain $\hat{w}_{i}$ explicitly, we show in Theorem 4.12 that this can be carried out for a particular choice of 
$T$ (which probably is not optimal). This permits us to obtain a family of admissible direction, depending on the subspace $W$ chosen. Essentially, they are of the form $B-A$ plus a term which has a growth of order two in $B-A$ for every $B \in M(\Omega)$. When the dimension of $W$ is equal to 1 , the corresponding admissible direction comes just from a lamination. In this case the expression of $H$ is known and it can be found, for example, in [25] (it can also be obtained from the results in [10]), but to our knowledge its utility to obtain optimality conditions for problem (1.1) has not been exploited. Most of the consequences we obtain in the present paper using Theorem 4.12 use only, in fact, $l=1$. However, we show that in some cases (see Remark 4.17) it is better to use a subspace of dimension greater than one. Using Theorem 4.12 we prove in Corollary 4.18 that for every $B \in M(\Omega)$, the condition

$$
C:(A-B) \geq 0
$$

(which is the condition we find if the admissible directions are of the form $B-A$ ) is still true on the set where $C$ has a nonpositive eigenvalue or where $\operatorname{Ker}(A-B) \neq\{0\}$. In particular (see Corollary 4.20) the condition (1.6) holds a.e. in $\Omega$ for every $B \in M(\Omega)$ when $k \leq N-1$. When $M(\Omega)$ comes from the mixture of a finite number of materials with fixed proportions, this result can also be obtained from the convexity of the set defined by (1.4), but we note that our set $M(\Omega)$ is more general.

In section 5 we study the case where $M(\Omega)$ is invariable by rotations, which is a natural assumption in the applications. Then we show that condition (1.3) implies that $C$ and $A$ are mutually diagonalizable a.e. in $\Omega$. Moreover, assuming further hypotheses (in particular if $M(\Omega)$ is $H$-closed and $N \geq 3$ ), we prove in Proposition 5.4 that the eigenvalues of $A$ and $C$ are mutually ordered.

As application of the results stated above, it is possible to obtain, in some situations, the matrix $A$ from $C$ and then to reduce the set of optimality conditions to a nonlinear partial differential system with variables $y_{i}, p_{i}, 1 \leq i \leq k$. The main problem to carrying out this point is that in general, the $H$-closure of a given set is unknown. In section 6, we apply our results to two examples: The first one is the mixture of two homogeneous isotropic materials, which has also been studied in [3] (see also [25] for $k=1$ ). In this case $M(\Omega)$ is convex. In second problem we consider a polycrystal in dimension 2 , where $M(\Omega)$ is not convex.

2. Notation. For a linear subspace $W \subset \mathbf{R}^{N}$, we define $\mathcal{L}(W, W)$ as the space of the linear applications from $W$ into $W$ and by $\mathcal{L}^{s}(W, W)$ the subspace of the symmetric applications. When $W=\mathbf{R}^{N}$ we write $\mathcal{M}_{N}=\mathcal{L}\left(\mathbf{R}^{N}, \mathbf{R}^{N}\right), \mathcal{M}_{N}^{s}=\mathcal{L}^{s}\left(\mathbf{R}^{N}, \mathbf{R}^{N}\right)$.

The orthogonal projection of $\mathbf{R}^{N}$ into $W$ is denoted by $P^{W}$.

For a matrix $A \in \mathcal{M}_{N}$, we define $A_{W} \in \mathcal{L}(W, W)$ by $A_{W}=P^{W} A_{\left.\right|_{W}}$.

The orthogonal subspace of $W$ is denoted by $W^{\perp}$.

For $u: W \rightarrow \mathbf{R}$, we denote $\nabla^{W} u: W \rightarrow W$ the gradient of $u$ with respect to $W$, i.e., $\nabla^{W} u$ is defined by

$$
\nabla^{W} u \xi=D_{\xi} u \quad \forall \xi \in W
$$

where $D_{\xi} u$ is the derivative of $u$ in the direction $\xi$.

We denote by $\left\{e_{1}, \ldots, e_{N}\right\}$ the standard basis of $\mathbf{R}^{N}$.

The group of the orthogonal matrices in $\mathbf{R}^{N}$ of determinant 1 is denoted by $\mathcal{O}_{N}$.

The scalar product of two matrices $A, B \in \mathcal{M}_{N}$ is written $A: B$.

The tensorial product of two vectors $\xi, \eta \in \mathbf{R}^{N}$ is denoted as $\xi \otimes \eta$. 
For a bounded open set $\Omega \subset \mathbf{R}^{N}$, we denote by $M(\Omega)$ a fixed subset of the space $L^{\infty}\left(\Omega, \mathcal{M}_{N}^{s}\right)$ such that there exist $\alpha, \beta>0$ which satisfy

$$
B(x) \xi \xi \geq \alpha|\xi|^{2}, \quad|B(x) \xi| \leq \beta|\xi| \quad \forall B \in M(\Omega) \text { a.e. } x \in \Omega .
$$

For a matrix $A \in M(\Omega), K_{A}(M(\Omega))$ and $\bar{K}_{A}(M(\Omega))$ are the cones of admissible directions of $M(\Omega)$ in $A$; see Definition 3.1.

For $T \subset \mathbf{R}^{N}$ and $\ell \in(0, N]$, we denote by $|T|_{\ell}$ the $\ell$-dimensional Hausdorff measure of $T$. The integral of a function $u: T \rightarrow \mathbf{R}$, with respect to the $\ell$-dimensional Hausdorff measure, is written

$$
\int_{T} u(z) d_{\ell} z
$$

When $\ell=N$, we simplify the notation by writing $|T|$ and

$$
\int_{T} u(z) d z
$$

We use the subindex $\sharp$ to mean periodicity. For example, for a cube $Y \subset \mathbf{R}^{N}$, $H_{\sharp}^{1}(Y)$ is the space of functions of $H_{l o c}^{1}\left(\mathbf{R}^{N}\right)$ which are $Y$-periodic.

3. Optimality conditions. In this section we introduce the definition of admissible direction. Using it, we obtain the first optimality result for the control problem (1.1).

Definition 3.1. For $A \in M(\Omega)$, let us define the cone of admissible directions $\bar{K}_{A}(M(\Omega))$ as the closure in the weak-* topology of $L^{\infty}\left(\Omega, \mathcal{M}_{N}^{s}\right)$ of the set $K_{A}(M(\Omega))$, where $K_{A}(M(\Omega))$ is the set of $H \in L^{\infty}\left(\Omega, \mathcal{M}_{N}^{s}\right)$ such that there exist a constant $c>0$ and $A_{\varepsilon} \in M(\Omega), \varepsilon>0$, such that

$$
\left\{\begin{array}{l}
\left\|A_{\varepsilon}-A\right\|_{L^{\infty}\left(\Omega, \mathcal{M}_{N}^{s}\right)} \leq c \varepsilon, \\
\lim _{\varepsilon \rightarrow 0} \frac{A_{\varepsilon}-A}{\varepsilon}=H \quad \text { a.e. in } \Omega .
\end{array}\right.
$$

Theorem 3.2. We consider $J: H_{0}^{1}(\Omega)^{k} \rightarrow \mathbf{R}$, Fréchet derivable, $f_{1}, \ldots, f_{k} \in$ $H^{-1}(\Omega)^{k}$. Let $A \in M(\Omega)$ be a solution of (1.1) and $y_{1}, \ldots, y_{k}$ the solutions of (1.2). We define the adjoint states $p_{1}, \ldots, p_{k}$ as the solutions of

$$
\begin{cases}-\operatorname{div}\left(A \nabla p_{i}\right)=\partial_{i} J\left(y_{1}, \ldots, y_{k}\right) & \text { in } \mathcal{D}^{\prime}(\Omega), \\ p_{i} \in H_{0}^{1}(\Omega), & 1 \leq i \leq k,\end{cases}
$$

and the matrix $C \in L^{1}\left(\Omega, \mathcal{M}_{N}^{s}\right)$ by

$$
C=\frac{1}{2} \sum_{i=1}^{k}\left(\nabla y_{i} \otimes \nabla p_{i}+\nabla p_{i} \otimes \nabla y_{i}\right) .
$$

Then we have

$$
\int_{\Omega} H: C d x \leq 0 \quad \forall H \in \bar{K}_{A}(M(\Omega)) .
$$


Proof. Let us first prove the result for $H \in K_{A}(M(\Omega))$. For $\varepsilon>0$ small enough, we define $y_{i, \varepsilon}^{*}, 1 \leq i \leq k$, as the solution of

$$
\left\{\begin{array}{l}
-\operatorname{div}\left((A+\varepsilon H) \nabla y_{i, \varepsilon}^{*}\right)=f_{i} \quad \text { in } \mathcal{D}^{\prime}(\Omega) \\
y_{i, \varepsilon}^{*} \in H_{0}^{1}(\Omega)
\end{array}\right.
$$

Then it is easy to check that for $1 \leq i \leq k$, we have

$$
\lim _{\varepsilon \rightarrow 0} \frac{y_{i, \varepsilon}^{*}-y_{i}}{\varepsilon}=\dot{y}_{i} \quad \text { in } H_{0}^{1}(\Omega)
$$

with $\dot{y}_{i}$ the solutions of

$$
\left\{\begin{array}{l}
-\operatorname{div}\left(A \nabla \dot{y}_{i}+H \nabla y_{i}\right)=0 \quad \text { in } \mathcal{D}^{\prime}(\Omega) \\
\dot{y}_{i} \in H_{0}^{1}(\Omega)
\end{array}\right.
$$

Now, for $\varepsilon>0$, we consider $A_{\varepsilon} \in M(\Omega)$ in the conditions of (3.1). Then for $1 \leq i \leq k$, we define $y_{i, \varepsilon}$ as the solutions of

$$
\left\{\begin{array}{l}
-\operatorname{div}\left(A_{\varepsilon} \nabla y_{i, \varepsilon}\right)=f_{i} \quad \text { in } \mathcal{D}^{\prime}(\Omega) \\
y_{i, \varepsilon} \in H_{0}^{1}(\Omega)
\end{array}\right.
$$

Taking $y_{i, \varepsilon}^{*}-y_{i, \varepsilon}$ as test function in the difference of (3.5) and (3.8), and dividing by $\varepsilon$, we get

$$
\begin{aligned}
\frac{1}{\varepsilon} \int_{\Omega}( & A+\varepsilon H) \nabla\left(y_{i, \varepsilon}^{*}-y_{i, \varepsilon}\right) \nabla\left(y_{i, \varepsilon}^{*}-y_{i, \varepsilon}\right) d x \\
= & \int_{\Omega} \frac{A_{\varepsilon}-(A+\varepsilon H)}{\varepsilon} \nabla\left(y_{i, \varepsilon}-y_{i, \varepsilon}^{*}\right) \nabla\left(y_{i, \varepsilon}^{*}-y_{i, \varepsilon}\right) d x \\
& \quad+\int_{\Omega} \frac{A_{\varepsilon}-(A+\varepsilon H)}{\varepsilon} \nabla y_{i, \varepsilon}^{*} \nabla\left(y_{i, \varepsilon}^{*}-y_{i, \varepsilon}\right) d x .
\end{aligned}
$$

By the ellipticity of $A+\varepsilon H$ (for $\varepsilon$ small enough) and (3.1), we deduce from (3.9) the existence of $c>0$ such that

$$
\frac{1}{\varepsilon}\left\|y_{i, \varepsilon}^{*}-y_{i, \varepsilon}\right\|_{H_{0}^{1}(\Omega)}^{2} \leq c\left\|y_{i, \varepsilon}^{*}-y_{i, \varepsilon}\right\|_{H_{0}^{1}(\Omega)}\left\|\mu_{\varepsilon}\right\|_{L^{2}(\Omega)},
$$

with

$$
\mu_{\varepsilon}=\frac{A_{\varepsilon}-(A+\varepsilon H)}{\varepsilon} \nabla y_{i, \varepsilon}^{*}
$$

From (3.1), (3.6), and the Lebesgue-dominated convergence theorem, we deduce that $\mu_{\varepsilon}$ converges strongly to zero in $L^{2}(\Omega)^{N}$. Thus,

$$
\lim _{\varepsilon \rightarrow 0} \frac{y_{i, \varepsilon}^{*}-y_{i, \varepsilon}}{\varepsilon}=0 \quad \text { in } H_{0}^{1}(\Omega)
$$

which, by (3.6), implies

$$
\lim _{\varepsilon \rightarrow 0} \frac{y_{i, \varepsilon}-y_{i}}{\varepsilon}=\dot{y}_{i} \quad \text { in } H_{0}^{1}(\Omega) .
$$


On the other hand, since $A$ is a solution of (1.1) and $A_{\varepsilon} \in M(\Omega)$, we have

$$
\frac{J\left(y_{\varepsilon}\right)-J(y)}{\varepsilon} \geq 0 \quad \forall \varepsilon>0
$$

with $y_{\varepsilon}=\left(y_{1, \varepsilon}, \ldots, y_{k, \varepsilon}\right)$ and $y=\left(y_{1}, \ldots, y_{k}\right)$. From (3.10) and the Fréchet derivability of $J$, we get

$$
\sum_{i=1}^{k}\left\langle\partial_{i} J(y), \dot{y}_{i}\right\rangle=\lim _{\varepsilon \rightarrow 0} \frac{J\left(y_{\varepsilon}\right)-J(y)}{\varepsilon} \geq 0 .
$$

But taking $\dot{y}_{i}$ as the test function in (3.2) and $p_{i}$ as the test function in (3.7), we have

$$
\sum_{i=1}^{k}\left\langle\partial_{i} J(y), \dot{y}_{i}\right\rangle=\sum_{i=1}^{k} \int_{\Omega} A \nabla p_{i} \nabla \dot{y}_{i} d x=-\sum_{i=1}^{k} \int_{\Omega} H \nabla y_{i} \nabla p_{i} d x .
$$

This proves

$$
\int_{\Omega} H: C d x=\sum_{i=1}^{k} \int_{\Omega} H \nabla y_{i} \nabla p_{i} d x \leq 0 \quad \forall H \in K_{A}(M(\Omega)) .
$$

Now let $H$ be in $\bar{K}_{A}(M(\Omega))$. For $\delta>0$ we define

$$
G_{\delta}=\left\{M \in L^{\infty}\left(\Omega, \mathcal{M}_{N}^{s}\right) /\left|\int_{\Omega} C:(M-H) d x\right|<\delta\right\} .
$$

Since $G_{\delta}$ is a neighborhood of $H$ in the weak-* topology of $L^{\infty}\left(\Omega, \mathcal{M}_{N}^{s}\right)$, there exists $H_{\delta}$ in $G_{\delta} \cap K_{A}(M(\Omega))$ and then, from (3.12), we get

$$
\int_{\Omega} C: H d x=\int_{\Omega} C: H_{\delta} d x+\int_{\Omega} C:\left(H-H_{\delta}\right) d x<\delta
$$

for every $\delta>0$. This proves (3.4).

Remark 3.3. The above theorem is still true if the elements of $M(\Omega)$ are not necessarily symmetric by changing $A$ to $A^{t}$ in the definition (3.2) of the functions $p_{i}$ and taking

$$
C=\sum_{i=1}^{k} \nabla p_{i} \otimes \nabla y_{i}
$$

Remark 3.4. If $M(\Omega)$ is convex, the condition (3.4) implies

$$
\int_{\Omega} A: C d x=\max \left\{\int_{\Omega} B: C d x / B \in M(\Omega)\right\} .
$$

Remark 3.5. Theorem 3.2 still holds if we take $K_{A}(M(\Omega))$ as the cone of matrices $H \in L^{\infty}\left(\Omega, \mathcal{M}_{N}^{s}(\Omega)\right)$ such that for every sequence $\Phi_{\varepsilon}^{1}, \ldots, \Phi_{\varepsilon}^{k}$, which respectively converges in $L^{2}(\Omega)^{N}$ to $\Phi^{1}, \ldots, \Phi^{k}$, there exists $A_{\varepsilon} \in M(\Omega)$ such that

$$
\frac{A_{\varepsilon}-A}{\varepsilon} \Phi_{\varepsilon}^{i} \rightarrow H \Phi^{i} \quad \text { in } L^{2}(\Omega)^{N}, \quad i=1, \ldots, k .
$$


The advantage of this definition is the following: If $M(\Omega)$ is the set we obtain by mixing $r$ materials with proportions fixed, then for every $\xi_{1}, \ldots, \xi_{N-1} \in \mathbf{R}^{N}$, the set

$$
\left\{\left(B \xi_{1}, \ldots, B \xi_{N-1}\right) / B \in M(\Omega)\right\} \subset L^{\infty}(\Omega)^{N-1}
$$

is convex (see [24], [25]). So, with this definition of $K_{A}(M(\Omega)$ ), the matrices of the form $B-A$ belong to $K_{A}(M(\Omega))$ if $k \leq N-1$. Thus, (3.13) still holds in this case. Later we will deduce this result (see Corollary 4.20) for more general choices of $M(\Omega)$, using simply Definition 3.1 of admissible directions.

4. Calculus of admissible directions. In the following, let us calculate explicitely some admissible directions by imposing additional hypotheses about $M(\Omega)$.

DeFinition 4.1. We say that $M(\Omega)$ is local if there exists a multivaluated application $F: x \in \Omega \rightarrow F(x) \subset \mathcal{M}_{N}^{s}$ such that

$$
M(\Omega)=\left\{B \in L^{\infty}\left(\Omega, \mathcal{M}_{N}^{s}\right) / B(x) \in F(x) \text { a.e. } x \in \Omega\right\},
$$

where $F$ is measurable in the sense that

$$
\{x \in \Omega / F(x) \cap G \neq \emptyset\} \text { is measurable } \forall G \subset \mathcal{M}_{N}^{s} \text { open. }
$$

As it is proved in [21], the local property is satisfied in several typical examples of $M(\Omega)$. A first consequence of assuming $M(\Omega)$ is local follows.

Proposition 4.2. We assume $M(\Omega)$ is local. We consider $A \in M(\Omega), H_{1}, \ldots$, $H_{m} \in K_{A}(M(\Omega)), \omega_{1}, \ldots, \omega_{m} \subset \Omega$ measurable such that $\left|\omega_{i} \cap \omega_{j}\right|=0$ if $i \neq j$. Then the matrix $H=\sum_{i=1}^{m} H_{i} \chi_{\omega_{i}}$ belongs to $K_{A}(M(\Omega))$.

Proof. By Definition 3.1, for every $i \in\{1, \ldots, m\}$ there exists $A_{\varepsilon}^{i} \in M(\Omega)$ and $c>0$ (which can be taken independent of $i$ ) such that

$$
\left\|A_{\varepsilon}^{i}-A\right\|_{L^{\infty}\left(\Omega, \mathcal{M}_{N}^{s}\right)} \leq c \varepsilon, \quad \lim _{\varepsilon \rightarrow 0} \frac{A_{\varepsilon}^{i}-A}{\varepsilon}=H_{i} \quad \text { a.e. in } \Omega .
$$

Taking then

$$
A_{\varepsilon}=\sum_{i=1}^{m} A_{\varepsilon}^{i} \chi_{\omega_{i}}+A \chi_{\Omega \backslash \cup_{i=1}^{m} \omega_{i}},
$$

which belongs to $M(\Omega)$ because $M(\Omega)$ is local, we have

$$
\left\|A_{\varepsilon}-A\right\|_{L^{\infty}\left(\Omega, \mathcal{M}_{N}^{s}\right)} \leq c \varepsilon, \quad \lim _{\varepsilon \rightarrow 0} \frac{A_{\varepsilon}-A}{\varepsilon}=H \quad \text { a.e. in } \Omega
$$

and then $H$ belongs to $K_{A}(M(\Omega))$.

Remark 4.3. It is not difficult to show that the above result remains true if we replace $K_{A}(M(\Omega))$ by $\bar{K}_{A}(M(\Omega))$.

Using Proposition 4.2, we get the following.

Proposition 4.4. In the assumptions of Theorem 3.2, if $M(\Omega)$ is local, we have

$$
H: C \leq 0 \quad \text { a.e. in } \Omega \forall H \in \bar{K}_{A}(M(\Omega)) .
$$

Proof. By Proposition 4.2, for every $H \in K_{A}(M(\Omega))$ and every $\omega \subset \Omega$ measurable, the matrix $H \chi_{\omega}$ belongs to $K_{A}(M(\Omega))$. So, using (3.4), we get

$$
\int_{\omega} H: C d x \leq 0
$$


Since $\bar{K}_{A}(M(\Omega))$ is the closure of $K_{A}(M(\Omega))$ in the weak-* topology of $L^{\infty}\left(\Omega, \mathcal{M}_{N}^{s}\right)$, we deduce that (4.2) holds, in fact, for every $H \in \bar{K}_{A}(M(\Omega))$ and every $\omega \subset \Omega$ measurable, which implies (4.1).

Let us now see how assuming $M(\Omega)$ is local permits us to obtain admissible directions.

THEOREM 4.5. We suppose that $M(\Omega)$ is local and closed for the $H$-convergence. We consider a linear subspace $W \subset \mathbf{R}^{N}$ of dimension $\ell$ and a measurable bounded subset $T \subset W$ such that $|T|_{\ell}$ is positive. Then, for every $A, B \in M(\Omega)$, the matrix $H \in L^{\infty}\left(\Omega, \mathcal{M}_{N}^{s}\right)$ defined by

$$
H(x) e_{i}=(B(x)-A(x))\left(e_{i}+\frac{1}{|T|_{\ell}} \int_{T} \nabla_{z}^{W} \hat{w}_{i}(x, z) d_{\ell}(z)\right)
$$

for $1 \leq i \leq N$ and a.e. $x \in \Omega$ belongs to $K_{A}\left(M(\Omega)\right.$ ). In (4.3), the function $\hat{w}_{i}$ is defined by

$$
\left\{\begin{array}{l}
\hat{w}_{i}(x, .) \in H_{l o c}^{1}(W), \quad \nabla_{z}^{W} \hat{w}_{i}(x, .) \in L^{2}(W, W), \\
\int_{W}\left(A(x) \chi_{W \backslash T}+B(x) \chi_{T}\right) \nabla_{z}^{W} \hat{w}_{i}(x, .) \nabla_{z}^{W} \hat{v} d_{\ell}(z) \\
\quad=\int_{T}(A(x)-B(x)) e_{i} \nabla_{z}^{W} \hat{v} d_{\ell}(z), \\
\forall \hat{v} \in H_{l o c}^{1}(W), \quad \nabla_{z}^{W} \hat{v} \in L^{2}(W, W) \quad \text { a.e. } x \in \Omega .
\end{array}\right.
$$

Proof. We consider $A, B, W$, and $T$ as in the statement of the theorem. For an orthonormal basis $\left\{e_{1}^{\prime}, \ldots, e_{\ell}^{\prime}\right\}$ of $W$, we denote

$$
Y=\left\{\sum_{i=1}^{\ell} \lambda_{i} e_{i}^{\prime} /-\frac{1}{2}<\lambda_{i}<\frac{1}{2}, 1 \leq i \leq \ell\right\} \subset W .
$$

For $\varepsilon>0$ small enough, we denote $T_{\varepsilon}=\varepsilon^{\frac{1}{\ell}} T \subset Y, \tilde{T}_{\varepsilon}=\bigcup_{k \in \mathbf{Z}^{\ell}}\left(T_{\varepsilon}+\sum_{i=1}^{\ell} k_{i} e_{i}^{\prime}\right)$, and we define $\tilde{A}_{\varepsilon}: \Omega \times W \rightarrow \mathcal{M}_{N}^{s}$ by

$$
\tilde{A}_{\varepsilon}(x, y)=A(x)\left(1-\chi_{\tilde{T}_{\varepsilon}}(y)\right)+B(x) \chi_{\tilde{T}_{\varepsilon}}(y) .
$$

Since $M(\Omega)$ is local and closed for the $H$-convergence, the matrix $A_{\varepsilon}$ obtained by taking, for $\varepsilon$ fixed, the $H$-limit when $\delta$ tends to zero of the matrices $x \rightarrow \tilde{A}_{\varepsilon}\left(x, \frac{1}{\delta} P^{W}(x)\right)$ belongs to $M(\Omega)$. Since the matrices $\tilde{A}_{\varepsilon}$ are a tensorial product of functions which only depend on $x$ and functions which only depend on $y$, it is well known (see, e.g., [5], [2]) that $A_{\varepsilon}$ is given by

$$
A_{\varepsilon}(x) e_{i}=\int_{Y} \tilde{A}_{\varepsilon}(x, y)\left(\nabla_{y}^{W} w_{i, \varepsilon}+e_{i}\right) d_{\ell}(y)
$$

where $w_{i, \varepsilon}$ is the unique solution of

$$
\left\{\begin{array}{l}
w_{i, \varepsilon} \in L^{2}\left(\Omega, H_{\sharp}^{1}(Y) / \mathbf{R}\right), \\
\int_{Y} \tilde{A}_{\varepsilon}(x, y)\left(\nabla_{y}^{W} w_{i, \varepsilon}(x, y)+e_{i}\right) \nabla_{y}^{W} v(y) d_{\ell}(y)=0 \\
\forall v \in H_{\sharp}^{1}(Y) / \mathbf{R} \quad \text { a.e. } x \in \Omega .
\end{array}\right.
$$


Let us study the asymptotic behavior of $A_{\varepsilon}$. First, we remark that for every $v \in$ $H_{\sharp}^{1}(Y) / \mathbf{R}$ and a.e. $x \in \Omega$, we have

$$
\begin{aligned}
\int_{Y} \tilde{A}_{\varepsilon}(x, y) e_{i} \nabla_{y}^{W} v(y) d_{\ell}(y)= & \int_{Y} A(x) e_{i} \nabla_{y}^{W} v(y) d_{\ell}(y) \\
& +\int_{T_{\varepsilon}}(B(x)-A(x)) e_{i} \nabla_{y}^{W} v(y) d_{\ell}(y),
\end{aligned}
$$

but for a.e. $x \in \Omega$, the first term on the right-hand side vanishes. So, $w_{i, \varepsilon}$ satisfies

$$
\left\{\begin{array}{c}
\int_{Y} \tilde{A}_{\varepsilon}(x, y) \nabla_{y}^{W} w_{i, \varepsilon}(x, y) \nabla_{y}^{W} v(y) d_{\ell}(y) \\
\quad=\int_{T_{\varepsilon}}(A(x)-B(x)) e_{i} \nabla_{y}^{W} v(y) d_{\ell}(y) \\
\forall v \in H_{\sharp}^{1}(Y) / \mathbf{R} \quad \text { a.e. } x \in \Omega .
\end{array}\right.
$$

For $1 \leq i \leq N$, we take $w_{i, \varepsilon}$ as the test function in (4.7). Then we get

$$
\int_{Y} \tilde{A}_{\varepsilon}(x, y) \nabla_{y}^{W} w_{i, \varepsilon}(x, y) \nabla_{y}^{W} w_{i, \varepsilon}(x, y) d_{\ell}(y)=\int_{T_{\varepsilon}}(A(x)-B(x)) e_{i} \nabla_{y}^{W} w_{i, \varepsilon}(x, y) d_{\ell}(y)
$$

for a.e. $x \in \Omega$. Using then (2.1) and the Cauchy-Schwarz inequality, we deduce there exists $c>0$ such that

$$
\int_{Y}\left|\nabla_{y}^{W} w_{i, \varepsilon}(x, y)\right|^{2} d_{\ell}(y) \leq c\left|T_{\varepsilon}\right|_{\ell}=c \varepsilon
$$

for $\varepsilon>0$ small enough and a.e. $x \in \Omega$.

We define $\hat{w}_{i, \varepsilon}: \Omega \times\left(\varepsilon^{-\frac{1}{\ell}} Y\right) \rightarrow \mathbf{R}$ by

$$
\hat{w}_{i, \varepsilon}(x, z)=\varepsilon^{-\frac{1}{\ell}} w_{i, \varepsilon}\left(x, \varepsilon^{\frac{1}{\ell}} z\right) .
$$

From (4.8), we deduce that $\nabla_{z}^{W} \hat{w}_{i, \varepsilon} \chi_{\varepsilon^{-\frac{1}{\ell}} Y}$ is bounded in $L^{\infty}\left(\Omega, L^{2}(W, W)\right)$. So there exists a subsequence of $\varepsilon$, which we still denote by $\varepsilon$, which converges weak-* in $L^{\infty}\left(\Omega, L^{2}(W, W)\right)$. Since the curl of the limit is zero, it is the gradient of a function $\hat{w}_{i} \in L^{\infty}\left(\Omega, H_{l o c}^{1}(W)\right)$. Once we prove that $\hat{w}_{i}$ is the solution of (4.4), we conclude that the whole of the sequence converges.

We consider $\hat{v} \in \mathcal{D}(W)$ and $\varepsilon>0$ small enough, such that $\varepsilon^{\frac{1}{\ell}} \operatorname{supp}(\hat{v}) \subset Y$, then we define $v_{\varepsilon} \in H_{\sharp}^{1}(Y)$ by

$$
v_{\varepsilon}(y)=\varepsilon^{\frac{1}{\ell}} \hat{v}\left(\varepsilon^{-\frac{1}{\ell}} y\right) \quad \text { a.e. } y \in Y .
$$

Taking $v_{\varepsilon}$ as the test function in (4.7), using the change of variables $z=\varepsilon^{-\frac{1}{\ell}} y$, and integrating with respect to $x$ in a measurable set $\omega$, we get

$$
\begin{gathered}
\int_{\omega} \int_{\varepsilon^{-\frac{1}{\tau} Y}}\left(A(x) \chi_{W \backslash T}(z)+B(x) \chi_{T}(z)\right) \nabla_{z}^{W} \hat{w}_{i, \varepsilon}(x, z) \nabla_{z}^{W} \hat{v}(z) d_{\ell}(z) d x \\
=\int_{\omega} \int_{T}(A(x)-B(x)) e_{i} \nabla_{z}^{W} \hat{v}(z) d_{\ell}(z) d x .
\end{gathered}
$$

Passing to the limit in this equality and taking into account the arbitrariness of $\hat{v}$ and $\omega$, and the density of $\mathcal{D}\left(\mathbf{R}^{N}\right) / \mathbf{R}$ in the factor space of functions with gradient in $L^{2}\left(\mathbf{R}^{N}\right)$ over $\mathbf{R}$ (see, e.g., [9]), we show that $\hat{w}_{i}$ is the solution of (4.4) for $1 \leq i \leq N$. 
Let us now prove

$$
\nabla_{z}^{W} \hat{w}_{i, \varepsilon} \chi_{\varepsilon^{-\frac{1}{\ell} Y}} \rightarrow \nabla_{z}^{W} \hat{w}_{i} \quad \text { in } L^{2}\left(\Omega, L^{2}(W, W)\right)
$$

for $1 \leq i \leq N$. For this purpose, it is enough to take $w_{i, \varepsilon}$ as the test function in (4.7), to use the change of variables $z=\varepsilon^{-\frac{1}{\ell}} y$, to take $\hat{w}_{i}$ as the test function in (4.4), and to use that $\nabla_{z}^{W} \hat{w}_{i, \varepsilon} \chi_{\varepsilon^{-\frac{1}{\ell} Y}}$ converges to $\nabla_{z}^{W} \hat{w}_{i}$, weak-* in $L^{\infty}\left(\Omega, L^{2}(W, W)\right)$. This gives

$$
\begin{aligned}
& \int_{\Omega} \int_{\varepsilon-\frac{1}{\varepsilon} Y}\left(A \chi_{W \backslash T}+B \chi_{T}\right) \nabla_{z}^{W} \hat{w}_{i, \varepsilon} \nabla_{z}^{W} \hat{w}_{i, \varepsilon} d_{\ell}(z) d x \\
& \quad=\frac{1}{\varepsilon} \int_{\Omega} \int_{Y} \tilde{A}_{\varepsilon} \nabla_{y}^{W} w_{i, \varepsilon} \nabla_{y}^{W} w_{i, \varepsilon} d_{\ell}(y) d x=\frac{1}{\varepsilon} \int_{\Omega} \int_{T_{\varepsilon}}(A-B) e_{i} \nabla_{y}^{W} w_{i, \varepsilon} d_{\ell}(y) d x \\
& \quad=\int_{\Omega} \int_{T}(A-B) e_{i} \nabla_{z}^{W} \hat{w}_{i, \varepsilon} d_{\ell}(z) d x \rightarrow \int_{\Omega} \int_{T}(A-B) e_{i} \nabla_{z}^{W} \hat{w}_{i} d_{\ell}(z) d x \\
& \quad=\int_{\Omega} \int_{W}\left(A \chi_{W \backslash T}+B \chi_{T}\right) \nabla_{z}^{W} \hat{w}_{i} \nabla_{z}^{W} \hat{w}_{i} d_{\ell}(z) d x .
\end{aligned}
$$

This implies (4.9).

Now, for $i, j \in\{1, \ldots, N\}$ and a.e. $x \in \Omega$, we write

$$
\begin{aligned}
A_{\varepsilon}(x) e_{i} e_{j} & =\int_{Y} \tilde{A}_{\varepsilon}(x, y)\left[\nabla_{y}^{W} w_{i, \varepsilon}+e_{i}\right] e_{j} d_{\ell}(y) \\
& =\int_{Y} \tilde{A}_{\varepsilon}(x, y) \nabla_{y}^{W} w_{i, \varepsilon} e_{j} d_{\ell}(y)+A(x) e_{i} e_{j}+\varepsilon|T|_{l}(B(x)-A(x)) e_{i} e_{j} .
\end{aligned}
$$

Taking $w_{i, \varepsilon}$ as the test function in the problem satisfied by $w_{j, \varepsilon}$ and using the change of variables $z=\varepsilon^{-\frac{1}{\ell}} y$, we have

$$
\begin{aligned}
& \int_{Y} \tilde{A}_{\varepsilon}(x, y) \nabla_{y}^{W} w_{i, \varepsilon} e_{j} d_{\ell}(y)=-\int_{Y} \tilde{A}_{\varepsilon}(x, y) \nabla_{y}^{W} w_{j, \varepsilon} \nabla_{y}^{W} w_{i, \varepsilon} d_{\ell}(y) \\
& \quad=-\varepsilon \int_{\varepsilon^{-\frac{1}{\ell}} Y}\left(A(x) \chi_{W \backslash T}(z)+B(x) \chi_{T}(z)\right) \nabla_{z}^{W} \hat{w}_{j, \varepsilon} \nabla_{z}^{W} \hat{w}_{i, \varepsilon} d_{\ell}(z) .
\end{aligned}
$$

So, from (4.10), we get

$$
\begin{aligned}
\left(\frac{A_{\varepsilon}-A}{\varepsilon}\right) e_{i} e_{j}= & |T|_{\ell}(B(x)-A(x)) e_{i} e_{j} \\
& -\int_{\varepsilon^{-\frac{1}{\ell} Y}}\left(A(x) \chi_{W \backslash T}(z)+B(x) \chi_{T}(z)\right) \nabla_{z}^{W} \hat{w}_{j, \varepsilon} \nabla_{z}^{W} \hat{w}_{i, \varepsilon} d_{\ell}(z) .
\end{aligned}
$$

Since $\nabla_{z}^{W} \hat{w}_{j, \varepsilon}, \nabla_{z}^{W} \hat{w}_{i, \varepsilon}$ are bounded in $L^{\infty}\left(\Omega, L^{2}(W, W)\right)$, we deduce that $\frac{A_{\varepsilon}-A}{\varepsilon}$ is bounded in $L^{\infty}\left(\Omega, \mathcal{M}_{N}^{s}\right)$. On the other hand, using (4.9), we have

$$
\begin{aligned}
& \lim _{\varepsilon \rightarrow 0} \int_{\varepsilon^{-\frac{1}{\ell}} Y}\left(A(x) \chi_{W \backslash T}(z)+B(x) \chi_{T}(z)\right) \nabla_{z}^{W} \hat{w}_{j, \varepsilon} \nabla_{z}^{W} \hat{w}_{i, \varepsilon} d_{\ell}(z) \\
& \quad=\int_{W}\left(A(x) \chi_{W \backslash T}(z)+B(x) \chi_{T}(z)\right) \nabla_{z}^{W} \hat{w}_{j} \nabla_{z}^{W} \hat{w}_{i} d_{\ell}(z) \\
& \quad=-\int_{T}(B(x)-A(x)) \nabla_{z}^{W} \hat{w}_{i} e_{j} d_{\ell}(z)
\end{aligned}
$$


where we have used the problem satisfied by $\hat{w}_{j}$ and where the limit is taken in $L^{1}(\Omega)$. So we have proved that the matrix

$$
e_{i} \rightarrow|T|_{\ell}(B(x)-A(x))\left(e_{i}+\frac{1}{|T|_{\ell}} \int_{T} \nabla_{z}^{W} \hat{w}_{i}(x, z) d_{\ell}(z)\right)
$$

is in $K_{A}(M(\Omega))$. Since $K_{A}(M(\Omega))$ is a cone, we conclude the proof of the theorem.

Remark 4.6. Theorem 4.5 applies, for example, to the case where $M(\Omega)$ is the set of materials which can be obtained by homogenization, mixing $m$ materials with the prescribed volume. These sets usually appear in problems of optimal design. Some applications are given in the last section of the paper; see also, e.g., [3], [8], [13], [25], and references therein.

Remark 4.7. The method used in the proof of Theorem 4.5 to obtain admissible directions, which consists of putting an inclusion of a tensor $B$ in a background of tensor $A$, is a variation of the classical Weierstrass test. Related ideas have been used, for example, by K. A. Lurie (see [8] and references therein).

Remark 4.8. In Theorem 4.5 the expression of $H$ when $T$ is contained in a subspace $W$ of dimension $l$ can be obtained from the corresponding result to $W=\mathbf{R}^{N}$ (and then the case $W=\mathbf{R}^{N}$ can be consider as the most interesting one). It is enough to consider the matrix $H_{\varepsilon}$ corresponding to $W=\mathbf{R}^{N}$ and $T_{\varepsilon} \subset \mathbf{R}^{N}$ defined by

$$
T_{\varepsilon}=\left\{x+\varepsilon y: x \in B(0,1) \cap W^{\perp}, y \in T\right\}
$$

and then pass to the limit in $\varepsilon$. The proof of Theorem 4.5 given above has the advantage that we do not need to realize this second limit.

Remark 4.9. The expression (4.3) of the admissible direction $H$ has the difficulty that the function $\hat{w}_{i}$ is not explicit. However, as we said in the introduction, we think that it can be used, for example, to obtain a steepest descent direction. Then the function $\hat{w}_{i}$ can be calculated numerically. For this purpose we recall that by (3.11) in the proof of Theorem 3.2 we have

$$
J\left(y_{\varepsilon}\right) \sim J(y)+\varepsilon \sum_{i=1}^{k}\left\langle\partial_{i} J(y), \dot{y}_{i}\right\rangle=J(y)-\varepsilon \int_{\Omega} H: C d x
$$

and then, since $M(\Omega)$ is local, an idea to obtain the steepest direction is to maximize the product $H: C$ in the closure of the matrices $H$ given by (4.3). By Remark 4.8, it is enough to consider $W=\mathbf{R}^{N}$. We remark that the set of such $H$ is bounded and it is not difficult to show that its closure is convex and thus is essentially a ball (for some norm).

In Theorem 4.5, the set $T$ does not depend on $x \in \Omega$. Thanks to Proposition 4.2 we can, in fact, take $T$ depending on $x$. A result in this sense, which we use later in Theorem 4.12, is the following.

Lemma 4.10. Assume $M(\Omega)$ is local and closed for the $H$-convergence. We consider a linear subspace $W \subset \mathbf{R}^{N}$ of dimension $\ell$, a measurable bounded subset $T_{0} \subset W$, and a matrix $E \in L^{\infty}(\Omega, \mathcal{L}(W, W))$ which is invertible a.e. in $\Omega$ and such that $E^{-1}$ also belongs to $L^{\infty}\left(\Omega, \mathcal{L}(W, W)\right.$ ). Then, taking $T(x)=E(x) T_{0}$, for a.e. $x \in \Omega$, the matrix $H$ defined by (4.3) with $T=T(x)$ is in $\bar{K}_{A}(M(\Omega)$ ) for every $A, B \in M(\Omega)$.

Proof. For $E$ in the conditions of the lemma, it is well known that there exists a sequence $E_{n}=\sum_{j=1}^{m(n)} E_{j, n} \chi_{\omega_{j, n}}$ with $E_{j, n} \in \mathcal{L}(W, W), \omega_{j, n} \subset \Omega$ measurable, 
$\cup_{j=1}^{m(n)} \omega_{j, n}=\Omega, \omega_{j, n} \cap \omega_{l, n}=\emptyset$ if $l \neq j$, and such that $E_{n}$ converges strongly to $E$ in $L^{\infty}(\Omega, \mathcal{L}(W, W)$ ). If $n \in \mathbf{N}$ is large enough (denoting by $\|\cdots\|$ the norm in $\left.L^{\infty}(\Omega, \mathcal{L}(W, W))\right)$, we have $\left\|E_{n}-E\right\|<\left\|E^{-1}\right\|^{-1}$, and then $E_{n}$ is also invertible and satisfies

$$
\left\|E_{n}^{-1}-E^{-1}\right\| \leq \frac{\left\|E^{-1}\right\|\|E-E n\|}{\left\|E^{-1}\right\|^{-1}-\left\|E-E_{n}\right\|} .
$$

So $E_{n}^{-1}$ also converges to $E^{-1}$ in $L^{\infty}(\Omega, \mathcal{L}(W, W))$.

We now take $T_{n}(x)=E_{n}(x) T_{0}$ and we denote by $H_{n} \in L^{\infty}\left(\Omega, \mathcal{M}_{N}^{s}\right)$ the matrix defined by

$$
H_{n}(x) e_{i}=(B(x)-A(x))\left(e_{i}+\frac{1}{\left|T_{n}\right|_{\ell}} \int_{T_{n}} \nabla_{z}^{W} \hat{w}_{i, n}(x, z) d_{\ell}(z)\right),
$$

where, for $i \in\{1, \ldots, n\}, \hat{w}_{i, n}$ is the solution of

$$
\left\{\begin{array}{l}
\hat{w}_{i, n}(x, .) \in H_{l o c}^{1}(W), \quad \nabla_{z}^{W} \hat{w}_{i, n}(x, .) \in L^{2}(W, W), \\
\int_{W}\left(A \chi_{W \backslash T_{n}}+B \chi_{T_{n}}\right) \nabla_{z}^{W} \hat{w}_{i, n} \nabla_{z}^{W} \hat{v} d_{\ell}(z) \\
\quad=\int_{T_{n}}(A-B) e_{i} \nabla_{z}^{W} \hat{v} d_{\ell}(z) \\
\forall \hat{v} \in H_{l o c}^{1}(W), \quad \nabla_{z}^{W} \hat{v} \in L^{2}(W, W) \quad \text { a.e. } x \in \Omega .
\end{array}\right.
$$

From Theorem 4.5 and Proposition 4.2, this function belongs to $K_{A}(M(\Omega))$. Using the change of variables $\tilde{w}_{i, n}(\tilde{z})=w_{i, n}\left(E_{n} \tilde{z}\right)$, we deduce that $\tilde{w}_{i, n}$ is the solution of

$$
\left\{\begin{array}{l}
\tilde{w}_{i, n}(x, .) \in H_{l o c}^{1}(W), \quad \nabla_{\tilde{z}}^{W} \tilde{w}_{i, n}(x, .) \in L^{2}(W, W), \\
\int_{W}\left(E_{n}^{-1}\right)^{t}\left(A \chi_{W \backslash T_{0}}+B \chi_{T_{0}}\right) E_{n}^{-1} \nabla_{\tilde{z}}^{W} \tilde{w}_{i, n} \nabla_{\tilde{z}}^{W} \tilde{v} d_{\ell}(z) \\
\quad=\int_{T_{0}}\left(E_{n}^{-1}\right)^{t}(A-B) e_{i} \nabla_{\tilde{z}}^{W} \tilde{v} d_{\ell}(z) \\
\forall \tilde{v} \in H_{l o c}^{1}(W), \quad \nabla_{\tilde{z}}^{W} \tilde{v} \in L^{2}(W, W) \text { a.e. } x \in \Omega .
\end{array}\right.
$$

From the uniform convergence of $E_{n}^{-1}$ to $E^{-1}$, we easily deduce that $\nabla_{\tilde{z}}^{W} \tilde{w}_{i, n}$ converges a $\nabla_{\tilde{z}}^{W} \tilde{w}_{i}$ in $L^{\infty}\left(\Omega, L^{2}(W, W)\right)$, with $\tilde{w}_{i}$ the solution of

$$
\left\{\begin{array}{l}
\tilde{w}_{i}(x, .) \in H_{l o c}^{1}(W), \quad \nabla_{\tilde{z}}^{W} \tilde{w}_{i}(x, .) \in L^{2}(W, W), \\
\int_{W}\left(E^{-1}\right)^{t}\left(A \chi_{W \backslash T_{0}}+B \chi_{T_{0}}\right) E^{-1} \nabla_{\tilde{z}}^{W} \tilde{w}_{i} \nabla_{\tilde{z}}^{W} \tilde{v} d_{\ell}(z) \\
\quad=\int_{T_{0}}\left(E^{-1}\right)^{t}(A-B) e_{i} \nabla_{\tilde{z}}^{W} \tilde{v} d_{\ell}(z) \\
\forall \tilde{v} \in H_{l o c}^{1}(W), \quad \nabla_{\tilde{z}}^{W} \tilde{v} \in L^{2}(W, W) \quad \text { a.e. } x \in \Omega .
\end{array}\right.
$$

Returning to the old variables, we then deduce that $\nabla_{z}^{W} \hat{w}_{i, n}$ converges to $\nabla_{z}^{W} \hat{w}_{i}$ in $L^{\infty}\left(\Omega, L^{2}(W, W)\right)$, with $\hat{w}_{i}$ the solution of (4.4). Thus, $H_{n}$ converges strongly to $H$ in $L^{\infty}\left(\Omega, \mathcal{M}_{N}^{s}\right)$. So $H$ belongs to $\bar{K}_{A}(M(\Omega))$.

Let us now obtain the solutions $\hat{w}_{i}$ of (4.4) for some particular choices of $T$ and then use Proposition 4.4 to obtain explicit optimality conditions. 
LEMma 4.11. If $M(\Omega)$ is local and closed for the $H$-convergence, then, for every linear subspace $W \subset \mathbf{R}^{N}$ of dimension $\ell$ and every $A, B \in M(\Omega)$, the matrix

$$
(B-A)-(B-A)(B+(\ell-1) A)_{W}^{-1} P^{W}(B-A)
$$

is in $\bar{K}_{A}(M(\Omega))$.

Proof. Let $W$ be a linear subspace of $\mathbf{R}^{N}$ of dimension $l$. Since $A$ and $A^{-1}$ are in $L^{\infty}\left(\Omega, \mathcal{M}_{N}^{s}\right)$, there exists $R \in L^{\infty}(\Omega, \mathcal{L}(W, W))$, with $R^{-1} \in L^{\infty}(\Omega, \mathcal{L}(W, W))$, such that $R A_{W} R^{t}=I_{W}$ a.e. in $\Omega$. We define $T_{0}$ the unitary ball in $W$ and for a.e. $x \in \Omega$ we take $T(x)=R(x)^{-1} T_{0}$ and $H(x)$ the matrix given by (4.3) with $T=T(x)$. From Lemma 4.10, $H$ belongs to $\bar{K}_{A}(M(\Omega))$.

The problem is to calculate the solution $\hat{w}_{i}, 1 \leq i \leq N$, of (4.4). For this purpose, the idea is to use the change of variables $z^{\prime}=R z$, which transforms (4.4) in a similar problem, where $A$ and $T$ are respectively replaced by the identity and the unitary ball in $W$. This problem can be solved by using in a suitable way the fundamental solution of the laplacian. Doing this and returning to the old variables we deduce that (up to a function which only depends on $x$ ) $\hat{w}_{i}$ is given by

$$
\hat{w}_{i}(x, z)=\left\{\begin{array}{ll}
\mu_{i}(x) z & \text { in } \mathrm{T}, \\
\frac{\mu_{i}(x) z}{|R z|^{\ell}} & \text { in } W \backslash T,
\end{array} \quad 1 \leq i \leq N,\right.
$$

where $\mu_{i}(x)=(B(x)+(\ell-1) A(x))_{W}^{-1} P^{W}(A(x)-B(x)) e_{i}$. Then by (4.3) we deduce

$$
H(x) e_{i}=(B(x)-A(x))\left(e_{i}+\mu_{i}(x)\right) .
$$

Taking into account the expression of $\mu_{i}$, we finish the proof of the theorem.

Using Lemma 4.11 and condition (3.4), we deduce the following theorem.

THEOREM 4.12. In the assumptions of Theorem 3.2, if $M(\Omega)$ is local and closed for the $H$-convergence, then, for every linear subspace $W \subset \mathbf{R}^{N}$ of dimension $\ell$, we have

$$
C:\left\{(A-B)+(A-B)(B+(\ell-1) A)_{W}^{-1} P^{W}(A-B)\right\} \geq 0 \quad \text { a.e. in } \Omega \forall B \in M(\Omega) \text {. }
$$

Remark 4.13. The condition (4.12) can also be written

$$
C:(A-B)+\min _{1 \leq \ell \leq N} \min _{\operatorname{dim}(W)=l} C:(A-B)(B+(\ell-1) A)_{W}^{-1} P^{W}(A-B) \geq 0 .
$$

Thus, the better choice for $W$ is to consider just the subspace which gives the minimum in this expression. This can also be related to the choice of the steepest descent direction, mentioned in Remark 4.9. If we restrict ourselves to the set of matrices $H$ of the form

$$
H=B-A-(A-B)(B+(\ell-1) A)_{W}^{-1} P^{W}(A-B),
$$

then to choose the matrix giving the maximum of $H: C$ is equivalent to solving the minimization problem which appears in (4.13).

Remark 4.14. The condition (4.12) must be compared with the usual one when $M(\Omega)$ is convex, which is

$$
C:(A-B) \geq 0 \quad \forall B \in M(\Omega), \text { a.e. in } \Omega .
$$


In general we get a perturbation of this condition with a term of second growth in $(B-A)$. We will see in Corollary 4.18 how condition (4.12) implies (4.14), at least in a subset of $\Omega$.

COROLLARY 4.15. In the assumptions of Theorem 3.2, if $M(\Omega)$ is local and closed for the $H$-convergence, then, for every $B \in M(\Omega)$, we have

$$
C:(A-B)+\frac{(A-B) C(A-B) \xi \xi}{B \xi \xi} \geq 0 \quad \forall \xi \in \mathbf{R}^{N} \backslash\{0\} \text { a.e. in } \Omega .
$$

Proof. It is enough, for $\xi \in \mathbf{R}^{N} \backslash 0$, to take $W=\{\lambda \xi / \lambda \in \mathbf{R}\}$ in Theorem 4.12 and to use that in this case:

$$
(A-B) B_{W}^{-1} P^{W}(A-B)=\frac{(A-B) \xi \otimes(A-B) \xi}{B \xi \xi} .
$$

Remark 4.16. The condition (4.15) is equivalent to

$$
C:(A-B)+\min _{B \xi \xi=1} B\left[B^{-1}(A-B) C(A-B)\right] \xi \xi \geq 0
$$

or, equivalently (observe that $B^{-1}(A-B) C(A-B)$ is symmetric with respect to the scalar product given by $(\xi \mid \eta)=B \xi \eta$ for every $\left.\xi, \eta \in \mathbf{R}^{N}\right)$,

$$
C:(A-B)+\min \left\{\lambda: \lambda \text { eigenvalue of } B^{-1}(A-B) C(A-B)\right\} \geq 0 .
$$

Remark 4.17. The inequality (4.15) has been obtained taking $\ell=1$ in (4.12); then it comes just from a lamination in the direction $\xi$. So Corollary 4.15 holds if we assume only $M(\Omega)$ is local and stable under lamination (and not necessarily by $H$ convergence). The sets of matrices stable under lamination have been characterized by Francfort and Milton in [10] and [15]. In particular, it has been shown that, under a suitable change of variables, the set $M(\Omega)$, assumed stable under lamination, is convex. Corollary 4.15 can also be obtained from this result. In fact, deriving the usual formula for the lamination of two matrices $A$ and $B$ in the direction $\xi$, it has been proved in [25] that the matrix

$$
B-A-\frac{(A-B) \xi \otimes(A-B) \xi}{B \xi \xi}
$$

is an admissible direction. However, this has not been applied in our knowledge to the obtaining of optimality conditions for problem (1.1). Most of the results we obtain in the following can be deduced using (4.15), and then one can conjecture that the choice $\ell=1$ is the best one in (4.12) (or even using all the matrices $H$ given by Theorem 4.5 ) or, equivalently (see Remark 4.13), that the minimum in (4.13) is attained for $\ell=1$. An easy counterexample shows that this is not true in general; it is enough to consider $C=B=I$ and $A=m I$ with $m>1$. Then the minimum of the eigenvalues of $B^{-1}(A-B) C(A-B)$ is $(m-1)^{2}$ while taking, for example, $W=\mathbf{R}^{N}$ we have

$$
\begin{aligned}
C: & (A-B)(B+(N-1) A)_{W}^{-1} P^{W}(A-B) \\
& =C:(A-B)(B+(N-1) A)^{-1}(A-B) \\
& =\frac{N(m-1)^{2}}{1+(N-1) m}<(m-1)^{2} .
\end{aligned}
$$


COROLLARY 4.18. In the assumptions of Theorem 3.2, with $M(\Omega)$ local and closed for the $H$-convergence, we define

$$
\Omega^{-}=\{x \in \Omega: \exists \lambda \leq 0 \text { eigenvalue of } C(x)\}
$$

and

$$
\Omega_{B}=\{x \in \Omega: \operatorname{Ker}(A(x)-B(x)) \neq\{0\}\} \quad \forall B \in M(\Omega) .
$$

Then we have

$$
C:(A-B) \geq 0 \quad \text { a.e. in } \Omega^{-} \cup \Omega_{B} \quad \forall B \in M(\Omega) .
$$

Proof. Let $B$ be in $M(\Omega)$. For a.e. $x \in \Omega_{B}$, we choose $\xi(x) \in \operatorname{Ker}(A(x)-$ $B(x)) \backslash\{0\}$, and for a.e. $x \in \Omega^{-} \backslash \Omega_{B}$, we take $e(x)$ as an eigenvector associated with a nonpositive eigenvalue of $C(x)$ and $\xi(x)=(A(x)-B(x))^{-1} e(x)$. Then, taking $\xi=\xi(x)$ in (4.15), we obtain (4.17).

By the above result, it is interesting to learn how many nonpositive eigenvalues have the matrix $C$. In this sense, we give the following theorem.

Theorem 4.19. For $\xi_{1}, \ldots, \xi_{k}, \eta_{1}, \ldots, \eta_{k} \in \mathbf{R}^{N} \backslash\{0\}$, we define

$$
\begin{gathered}
\phi_{i}^{+}=\frac{\xi_{i}}{\left|\xi_{i}\right|}+\frac{\eta_{i}}{\left|\eta_{i}\right|}, \quad \phi_{i}^{-}=\frac{\xi_{i}}{\left|\xi_{i}\right|}-\frac{\eta_{i}}{\left|\eta_{i}\right|}, \\
m=\operatorname{dim}\left(\operatorname{Span}\left\{\xi_{i}, \eta_{i} / 1 \leq i \leq k\right\}\right)=\operatorname{dim}\left(\operatorname{Span}\left\{\phi_{i}^{+}, \phi_{i}^{-} / 1 \leq i \leq k\right\}\right), \\
m^{+}=\operatorname{dim}\left(\operatorname{Span}\left\{\phi_{i}^{+} / 1 \leq i \leq k\right\}\right), \quad m^{-}=\operatorname{dim}\left(\operatorname{Span}\left\{\phi_{i}^{-} / 1 \leq i \leq k\right\}\right), \\
\tilde{C}_{i}=\frac{1}{2}\left(\xi_{i} \otimes \eta_{i}+\eta_{i} \otimes \xi_{i}\right), \quad 1 \leq i \leq k, \quad \tilde{C}=\sum_{i=1}^{k} \tilde{C}_{i} .
\end{gathered}
$$

Then we have the following:

(i) For $1 \leq i \leq k$, the matrix $\tilde{C}_{i}$ has as eigenvalues $\frac{1}{2}\left(\xi_{i} \eta_{i}+\left|\xi_{i}\right|\left|\eta_{i}\right|\right) \geq 0$, $\frac{1}{2}\left(\xi_{i} \eta_{i}-\left|\xi_{i}\right|\left|\eta_{i}\right|\right) \leq 0$, with respective eigenvectors $\phi_{i}^{+}, \phi_{i}^{-}$. The other eigenvalues of $\tilde{C}_{i}$ are zero.

(ii) If $k^{+}$and $k^{-}$are, respectively, the number of positive and negative eigenvalues of $\tilde{C}$, we have

$$
m-m^{-} \leq k^{+} \leq m^{+}, \quad m-m^{+} \leq k^{-} \leq m^{-} .
$$

Proof. The proof of (i) is easy to verify. In order to prove (ii), we use the Courant-Fischer characterization of the eigenvalues:

$$
\lambda_{i+1}=\max _{\operatorname{dim} E \leq i} \min _{\substack{\phi \in E^{\perp} \\|\phi|=1}} \tilde{C} \phi \phi,
$$

where $\lambda_{1} \leq \cdots \leq \lambda_{N}$ are the eigenvalues of $\tilde{C}$.

Taking $i=m^{-}$and $E=\operatorname{Span}\left\{\phi_{j}^{-} / 1 \leq j \leq k\right\}$, statement (i) gives

$$
\lambda_{m^{-}+1} \geq \min _{\substack{\phi \in E^{\perp} \\|\phi|=1}} \tilde{C} \phi \phi \geq 0 .
$$

So $k^{-} \leq m^{-}$. 
Now, if $m-m^{-}=0$, then clearly $k^{+} \geq m-m^{-}$. In another case, we consider $E=$ $\operatorname{Span}\left(\left\{\phi_{j}^{-} / 1 \leq j \leq k\right\} \cup\left\{\phi_{j}^{-}, \phi_{j}^{+} / 1 \leq j \leq k\right\}^{\perp}\right)$, which has dimension $m^{-}+N-m$. If $\phi \in E^{\perp}$, then, as above, statement (i) gives $\tilde{C}_{j} \phi \phi \geq 0,1 \leq j \leq k$, and then $\tilde{C} \phi \phi \geq 0$. Moreover, $\tilde{C} \phi \phi=0$ iff $\tilde{C}_{j} \phi \phi=0,1 \leq j \leq k$, which, by statement (i), implies that $\phi$ is orthogonal to $\phi_{j}^{-}, \phi_{j}^{+}, 1 \leq j \leq k$; i.e., $\phi$ is in $E$ and so $\phi=0$. Thus, taking $i=m^{-}+N-m$ in (4.19) and using the compactness of the unitary ball in $\mathbf{R}^{N}$, we get

$$
\lambda_{m^{-}+N-m+1} \geq \min _{\substack{\phi \in E^{\perp} \\|\phi|=1}} \tilde{C} \phi \phi>0
$$

and thus $k^{+} \geq m-m^{-}$.

The other inequalities in (4.18) follow analogously.

As a consequence we get the following.

COROLLARY 4.20. In the assumptions of Theorem 3.2, if $M(\Omega)$ is local and closed for the $H$-convergence and $k \leq N-1$, then condition (4.14) holds.

Proof. We apply Theorem 4.19 to $\xi_{i}=\nabla y_{i}(x), \eta_{i}=\nabla p_{i}(x), 1 \leq i \leq k$, a.e. $x \in \Omega$. In this case $\tilde{C}=C(x)$. Since, clearly, the number $m^{+}$which appears in this result is less than or equal to $k \leq N-1$, we deduce that the number of positive eigenvalues of $C$ is less than or equal to $N-1$, and then there exists at least a nonpositive eigenvalue of $C$ a.e. in $\Omega$. Corollary 4.18 gives then (4.14).

5. Invariability by rotations. In the applications, it is a natural hypothesis to assume that $M(\Omega)$ is invariable by rotations. We show in this section that this assumption implies that the eigenvectors of $A$ and $C$ agree.

DeFINITION 5.1. We say that $M(\Omega)$ is invariable by rotations if for every $B \in$ $M(\Omega)$ and every $Q \in L^{\infty}\left(\Omega, \mathcal{M}_{N}\right)$, with $Q \in \mathcal{O}_{N}$ a.e. in $\Omega$, the matrix $Q B Q^{t}$ belongs to $M(\Omega)$.

We have the following result.

Proposition 5.2. In the assumptions of Theorem 3.2, if $M(\Omega)$ is invariable by rotations, then $A$ and $C$ are mutually diagonalizable a.e. in $\Omega$.

Proof. Let us first prove that given a skew-symmetric matrix $R$ and a measurable set $\omega \subset \Omega$, the function $\left(R A+A R^{t}\right) \chi_{\omega}$ belongs to $K_{A}(M(\Omega))$. To this purpose we define $G: \mathcal{M}_{N} \rightarrow \mathcal{M}_{N} \times \mathbf{R}$ by $G(M)=\left(M M^{t}, \operatorname{det}(M)\right)$. Since $\operatorname{Ker}\left(G^{\prime}(I)\right)$ coincides with the space of skew-symmetric matrices, it is known (see, e.g., [1]) that for $\varepsilon \in \mathbf{R}$ with $|\varepsilon|$ small enough, there exists $P_{\varepsilon} \in \mathcal{M}_{N}$ such that $G\left(P_{\varepsilon}\right)=G(I)$ or, equivalently, $P_{\varepsilon} \in \mathcal{O}_{N}$, and $\left(P_{\varepsilon}-I\right) / \varepsilon$ converges to $R$. Defining then

$$
A_{\varepsilon}=P_{\varepsilon} A P_{\varepsilon}^{t} \chi_{\omega}+A \chi_{\Omega \backslash \omega}
$$

and using that $M(\Omega)$ is invariable by rotations, we deduce that $A_{\varepsilon}$ belongs to $M(\Omega)$ and $\left(A_{\varepsilon}-A\right) / \varepsilon$ converges to $\left(R A+A R^{t}\right) \chi_{\omega}$ in $L^{\infty}\left(\Omega, \mathcal{M}_{N}^{s}\right)$. Thus $\left(R A+A R^{t}\right) \chi_{\omega}$ belongs to $K_{A}(M(\Omega))$.

Using now that the set of skew-symmetric matrices is a vectorial space, condition (3.4), and the arbitrariness of $\omega$, we deduce

$$
2(R A): C=\left(R A+A R^{t}\right): C=0 \quad \text { a.e. in } \Omega .
$$

For $i, j \in\{1, \ldots, N\}, i \neq j$, we take in the above equation $R$ as the matrix 
defined by

$$
R_{l k}= \begin{cases}1 & \text { if } l=i, k=j \\ -1 & \text { if } l=j, k=i \\ 0 & \text { in another case }\end{cases}
$$

Then we get

$$
(A C)_{i j}-(C A)_{i j}=0 \quad \text { a.e. in } \Omega,
$$

i.e., $A$ and $C$ commute, and then they are mutually diagonalizable.

Remark 5.3. From Proposition 5.2, assuming that the matrix $C$ is known and that their eigenvalues are all different, we must look for the optimal solution $A$ on the set of matrices of $M(\Omega)$ which have the same eigenvectors as $C$ a.e. So it can be interesting to write condition (4.13) assuming that $B$ is also mutually diagonalizable with $C$. If we restrict ourselves to the spaces $W$ which are generated by eigenvectors of $C$, we get the following result: In the conditions of Proposition 5.2, if $c_{i}, a_{i}, i \in\{1, \ldots, N\}$, are, respectively, the eigenvalues of $C$ and $A$, then for every $B \in M(\Omega)$ mutually diagonalizable with $A$ and $C$, with eigenvalues $b_{1}, \ldots, b_{N}$, we have

$$
\sum_{i=1}^{N} c_{i}\left(a_{i}-b_{i}\right)+\min _{1 \leq \ell \leq N} \min _{1 \leq i_{1}<\cdots<i_{\ell} \leq N} \sum_{j=1}^{\ell} \frac{c_{i_{j}}\left(a_{i_{j}}-b_{i_{j}}\right)^{2}}{b_{i_{j}}+(\ell-1) a_{i_{j}}} \geq 0 .
$$

We also note that by Remark 4.16, for $A, B, C$ as above, the condition (5.1) implies in particular (4.15).

Assuming stronger hypotheses, we can improve Proposition 5.2. Proposition 5.4 below is related to a theorem due to Lewis [11], which applies to the optimization of a function $h: \mathcal{M}_{N}^{s} \rightarrow \mathbf{R}$ convex and invariable by rotations (see also [12], where there is a review of results corresponding to optimization problems on symmetric matrices).

Proposition 5.4. In the assumptions of Theorem 3.2, we assume $M(\Omega)$ invariable by rotations and at least one of the following hypotheses:

(i) $M(\Omega)$ is convex.

(ii) $M(\Omega)$ is $H$-closed and $N \geq 3$.

(iii) $M(\Omega)$ is $H$-closed, $N=2$ and $k=1$.

Then there exists $Q \in L^{\infty}\left(\Omega, \mathcal{M}_{N}\right)$, with $Q \in \mathcal{O}_{N}$ a.e. in $\Omega$, such that

$$
\begin{aligned}
& Q A Q^{t}=\operatorname{diag}\left(a_{1}, \ldots, a_{N}\right), \\
& Q C Q^{t}=\operatorname{diag}\left(c_{1}, \ldots, c_{N}\right),
\end{aligned}
$$

and $a_{1} \leq \cdots \leq a_{N}, c_{1} \leq \cdots \leq c_{N}$.

Proof. From Proposition 5.2 there exists $Q \in L^{\infty}\left(\Omega, \mathcal{M}_{N}\right)$, with $Q \in \mathcal{O}_{N}$ a.e. in $\Omega$, such that (5.2) holds. Clearly, we can also assume $c_{1} \leq \cdots \leq c_{N}$ a.e. in $\Omega$. We consider $i, j \in\{1, \ldots, N\}, i \neq j$, and we take $L \in \mathcal{O}_{N}$, defined by

$$
L e_{i}=e_{j}, \quad L e_{j}=-e_{i}, \quad L e_{l}=e_{l} \quad \forall l \neq i, j .
$$

Since $M(\Omega)$ is invariable by rotations, the matrix $B=(L Q)^{t} \operatorname{diag}\left(a_{1}, \ldots, a_{N}\right) L Q$ belongs to $M(\Omega)$. Let us now see that if one of the hypotheses (i), (ii) or (iii) hold, then

$$
C:(A-B) \geq 0 \quad \text { a.e. in } \Omega
$$


For this purpose, we define $\tilde{M}(\Omega)$ as the convex hull of

$$
\left\{S A S^{t} / S \in L^{\infty}\left(\Omega, \mathcal{M}_{N}\right), S \in \mathcal{O}_{N} \text { a.e. in } \Omega\right\}
$$

if (i) holds and as the $H$-closure of this set in cases (ii) and (iii). Then $\tilde{M}(\Omega)$ is contained in $M(\Omega)$ and $A$ belongs to $\tilde{M}(\Omega)$, so $A$ is also a solution of (1.1) with $M(\Omega)$ replaced by $\tilde{M}(\Omega)$.

In case (i), $\tilde{M}(\Omega)$ is convex and local, so from (4.1) and $B-A$ in $K_{A}(M(\Omega))$, we deduce (5.3).

In cases (ii) and (iii), $\tilde{M}(\Omega)$ is local and $H$-closed. Moreover, if (ii) holds, then $\operatorname{Ker}(B-A) \neq 0$ a.e. in $\Omega$, while if we have (iii) then, from Theorem $4.19, C$ has at least a nonpositive eigenvalue. So, in both situations, we deduce (5.3) from (4.17).

From (5.3) we get

$$
0 \leq C:(A-B)=c_{i}\left(a_{i}-a_{j}\right)+c_{j}\left(a_{j}-a_{i}\right)=\left(c_{i}-c_{j}\right)\left(a_{i}-a_{j}\right) \quad \text { a.e. in } \Omega .
$$

This finishes the proof of Proposition 5.4.

Remark 5.5. As we have seen in the proof of Proposition 5.4, the order relation between the eigenvalues of $C$ and $A$ is a consequence of (5.3) with $B$ defined as above. So if $N=2$ and $M(\Omega)$ is $H$-closed, by Corollary 4.18, the thesis of Proposition 5.4 still holds on the set where $C$ has a least a nonnegative eigenvalue. Where the two eigenvalues are positive, assuming $c_{1}<c_{2}$, the condition (4.15) implies

$$
a_{1}<a_{2} \quad \text { or } \quad a_{1}>a_{2} \quad \text { and } \quad c_{2} a_{2} \leq c_{1} a_{1}
$$

Related to this inequality, we also remark that if in the conditions of Proposition 5.4 (ii) there are two eigenvalues of $C, c_{i}, c_{j}$ such that $c_{i} \leq c_{j} \leq 0$, then besides $a_{i} \leq a_{j}$, we have $\left|c_{j}\right| a_{j} \leq\left|c_{i}\right| a_{i}$.

6. Applications. In this section let us show how the condition (3.4) and the consequences we have obtained from it can be used, in some cases, to obtain $A \nabla y_{i}$, $A \nabla p_{i}, 1 \leq i \leq k$, as explicit functions of $\nabla y_{i}, \nabla p_{i}$ and then, from (1.2) and (3.2), to reduce the optimality conditions given in Theorem 3.2 to a nonlinear system in $\nabla y_{i}$, $\nabla p_{i}$. The main difficulty in carrying out this idea is that obtaining the $H$-closure of a subset of $L^{\infty}\left(\Omega, \mathcal{M}_{N}\right)$ is a very difficult problem, which has only been solved in some particular cases (see [23], [14], [13], [8], [16], [25]). To simplify the exposition, we have chosen two simple problems where the $H$-closure is well known. The first consists of the mixture of two homogeneous isotropic materials in dimension two (the problem can also be studied analogously for higher dimensions). This problem has also been studied in [3] and [7]. In this case the set $M(\Omega)$ is convex. In the second problem we consider a nonconvex situation corresponding to a polycrystal in dimension two.

First problem. We start by recalling the following result which has been proved in [23] and [14].

Theorem 6.1. We assume $N=2$. For $0<\alpha \leq \beta$ and $\theta \in L^{\infty}(\Omega)$ with $0 \leq \theta \leq 1$ a.e. in $\Omega$, the set $\mathcal{M}_{\theta}(\Omega)$ of the $H$-limits of the sequences $\alpha I \chi_{\omega_{n}}+\beta\left(1-\chi_{\omega_{n}}\right) I$, such that $\omega_{n} \subset \Omega$ are measurable sets and satisfy $\chi_{\omega_{n}}$ converges weakly-* in $L^{\infty}(\Omega)$ to $\theta$, is characterized as follows:

$\mathcal{M}_{\theta} \subset L^{\infty}\left(\Omega, \mathcal{M}_{2}^{s}\right)$ is the set of matrices such that their eigenvalues $\lambda_{1}, \lambda_{2}$ satisfy 
the following inequalities a.e. in $\Omega$ :

$$
\left\{\begin{array}{l}
\lambda^{-} \leq \lambda_{1}, \lambda_{2} \leq \lambda^{+}, \\
\sum_{i=1}^{2} \frac{1}{\lambda_{i}-\alpha} \leq \frac{1}{\lambda^{-}-\alpha}+\frac{1}{\lambda^{+}-\alpha}, \\
\sum_{i=1}^{2} \frac{1}{\beta-\lambda_{i}} \leq \frac{1}{\beta-\lambda^{-}}+\frac{1}{\beta-\lambda^{+}},
\end{array}\right.
$$

where $\lambda^{-}, \lambda^{+}$are given by

$$
\lambda^{+}=\alpha \theta+\beta(1-\theta), \quad \lambda^{-}=\left(\frac{\theta}{\alpha}+\frac{1-\theta}{\beta}\right)^{-1} .
$$

Let us see how the results obtained in the previous sections permit us to obtain $A$ from $C$, and then from $\nabla y_{i}, \nabla p_{i}, 1 \leq i \leq k$, for some choices of $M(\Omega)$ related to $\mathcal{M}_{\theta}(\Omega)$.

In the following, we define

$$
\check{\Omega}=\left\{x \in \Omega /\left(c_{1}(x), c_{2}(x)\right) \neq(0,0)\right\},
$$

where $c_{1}, c_{2}$ are the eigenvalues of the matrix $C$.

Proposition 6.2. In the assumptions of Theorem 3.2, if $M(\Omega)$ is the set of matrices defined in Theorem 6.1 for a fixed function $\theta$ and $c_{1}, c_{2}, c_{1} \leq c_{2}$, are the eigenvalues of $C$, then there exists an associated basis $\left\{\mu_{1}, \mu_{2}\right\}$ of eigenvectors of $C$ such that a.e. in $\Omega$, we have

$$
A \mu_{i}=a_{i} \mu_{i}, \quad i=1,2,
$$

where a.e. in $\check{\Omega}$, the functions $a_{1}, a_{2}$ are given by the following:

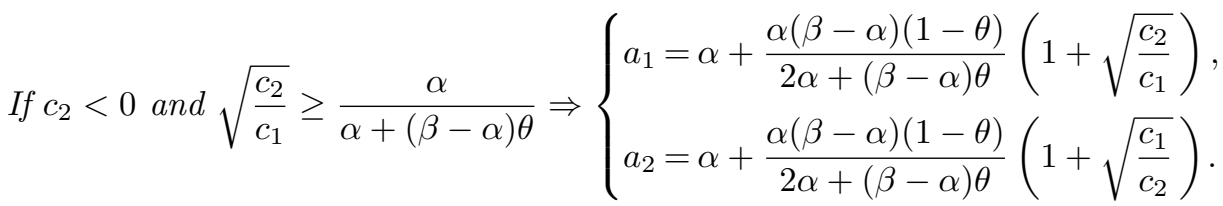

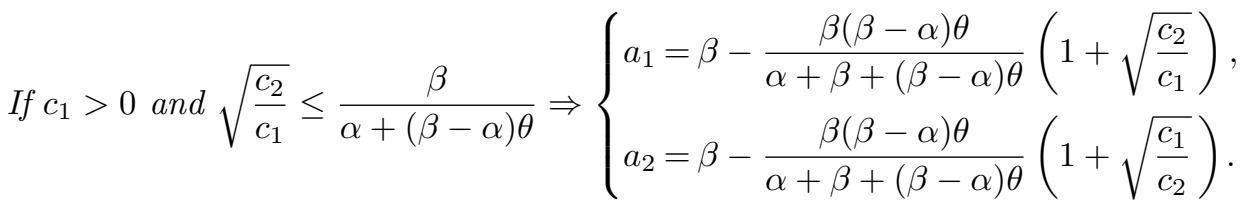

In another case $a_{1}=\lambda^{-}, a_{2}=\lambda^{+}$, where $\lambda^{-}, \lambda^{+}$are defined by (6.2).

Proof. In this case, the set $M(\Omega)$ is local, convex, and invariable by rotations. So we can apply Proposition 5.4 and (4.14), which imply that a.e. in $\Omega, A$ satisfies (6.4) for a basis of eigenvectors of $C$, and $a_{1} c_{1}+a_{2} c_{2}$ is the maximum of $\lambda_{1} c_{1}+\lambda_{2} c_{2}$, with $\lambda_{1}, \lambda_{2}$ in the set defined by (6.1). Solving this maximum problem we get the expressions $a_{1}$ and $a_{2}$ given in Proposition 6.2.

The above result assumes that $\theta$ is known, but clearly this is not a realistic situation. Next, we consider two examples where $\theta$ also varies. In the first one we impose the condition

$$
\frac{1}{|\Omega|} \int_{\Omega} \theta d x=s
$$


with $s \in(0,1)$. This means we know the proportion of the materials defined by $\alpha$ and $\beta$ but not its local distribution. This usually holds when one material is better than the other but it is also more expensive. In the second situation we consider the case where we do not have any restriction on $\theta$.

Proposition 6.3. In the assumptions of Theorem 3.2, if for $s \in(0,1)$ given, $M(\Omega)$ is the set of matrices defined in Theorem 6.1 , with $\theta \in L^{\infty}(\Omega), 0 \leq \theta \leq 1$ a.e. in $\Omega$ and such that (6.5) holds, then the matrix A satisfies the thesis of Proposition 6.2 , where the corresponding function $\theta$ is such that defining $F \in L^{\infty}(\check{\Omega})$ by

$$
F(x)= \begin{cases}\frac{\alpha\left(\beta^{2}-\alpha^{2}\right)\left(\sqrt{-c_{1}}-\sqrt{-c_{2}}\right)^{2}}{(2 \alpha+(\beta-\alpha) \theta)^{2}} & \text { if } c_{2}<0 \text { and } \sqrt{\frac{c_{2}}{c_{1}}} \geq \frac{\alpha}{\alpha+(\beta-\alpha) \theta}, \\ -\frac{\beta\left(\beta^{2}-\alpha^{2}\right)\left(\sqrt{c_{1}}+\sqrt{c_{2}}\right)^{2}}{(\alpha+\beta+(\beta-\alpha) \theta)^{2}} & \text { if } c_{1}>0 \text { and } \sqrt{\frac{c_{2}}{c_{1}}} \leq \frac{\beta}{\alpha+(\beta-\alpha) \theta}, \\ -(\beta-\alpha)\left(\frac{\alpha \beta}{(\alpha+(\beta-\alpha) \theta)^{2}} c_{1}+c_{2}\right) & \text { in another case, }\end{cases}
$$

there exists $r \in \mathbf{R}$ which satisfies

$$
\left\{\begin{array}{l}
F(x) \leq r \quad \text { a.e. in }\{\theta=0\} \cap \check{\Omega}, \\
F(x)=r \quad \text { a.e. in }\{0<\theta<1\} \cap \check{\Omega}, \\
F(x) \geq r \quad \text { a.e. in }\{\theta=1\} \cap \check{\Omega} .
\end{array}\right.
$$

Proof. We remark that if $\theta$ is the corresponding function associated with $A$ in the definition of the elements of $M(\Omega)$ and we define $M_{\theta}(\Omega)$ as in the statement of Theorem 6.1 , then $A$ is also the solution of (1.1) with $M(\Omega)$ replaced by $M_{\theta}(\Omega)$. So Proposition 6.2 applies.

Let us now vary $\theta$. For every $\theta^{*} \in L^{\infty}(\Omega)$ such that $0 \leq \theta^{*} \leq 1$ a.e. in $\Omega$,

$$
\frac{1}{|\Omega|} \int_{\Omega} \theta^{*} d x=s
$$

and $\theta^{*}=\theta$ a.e. in $\Omega \backslash \check{\Omega}$, we define $A_{\theta^{*}} \in M(\Omega)$ as the function given by Proposition 6.2 applied to $\theta^{*}$ a.e. in $\check{\Omega}$ and $A_{\theta^{*}}=A$ a.e. in $\Omega \backslash \check{\Omega}$. Deriving $A_{\theta^{*}}$ with respect to $\theta^{*}$, we obtain an admissible direction. Then, using condition (3.4), we get

$$
\int_{\Omega} F \vartheta d x \leq 0
$$

for every $\vartheta \in L^{\infty}(\Omega)$ such that $\vartheta=0$ a.e. in $\Omega \backslash \check{\Omega}, \vartheta \geq 0$ a.e. in $\{\theta=0\} \cap \check{\Omega}, \vartheta \leq 0$ a.e. in $\{\theta=1\} \cap \Omega$, and

$$
\int_{\Omega} \vartheta d x=0
$$

It is easy to check that this implies the existence of $r \in \mathbf{R}$, which satisfies the statement of the proposition.

Remark 6.4. The expression of $F$ is strictly decreasing with respect to $\theta$. Then, from (6.6), it is possible to obtain $\theta$ as a function of $c_{1}, c_{2}$ and $r$.

Remark 6.5. In Proposition 6.3, if $r \geq 0$, then $\theta=0$ a.e. in the set

$$
\left\{c_{1}>0\right\} \bigcap\left\{\sqrt{\frac{c_{2}}{c_{1}}} \leq \frac{\beta}{\alpha}\right\} \cap \check{\Omega} .
$$


Analogously, if $r \leq 0$, then $\theta=1$ a.e. in the set

$$
\left\{c_{2}<0\right\} \bigcap\left\{\sqrt{\frac{c_{2}}{c_{1}}} \geq \frac{\alpha}{\beta}\right\} \cap \check{\Omega} .
$$

We finish with the following result.

Proposition 6.6. In the assumptions of Theorem 3.2, let $M(\Omega)$ be the set of matrices defined in Theorem 6.1, where $\theta$ is any function in $L^{\infty}(\Omega)$ such that $0 \leq \theta \leq 1$ a.e. in $\Omega$, and denote by $c_{1}, c_{2}, c_{1} \leq c_{2}$, the eigenvalues of $C$. Then there exists an associated basis $\left\{\mu_{1}, \mu_{2}\right\}$ of eigenvectors of $C$ such that a.e. in $\Omega$ we have (6.4). Moreover, a.e. in $\check{\Omega}$, the functions $a_{1}, a_{2}$ are given by the following:

$$
\begin{aligned}
& \text { If } 0 \leq c_{1} \leq c_{2} \text {, then } a_{1}=a_{2}=\beta . \\
& \text { If } c_{1} \leq c_{2} \leq 0 \text {, then } a_{1}=a_{2}=\alpha . \\
& \text { If } c_{1}<0<c_{2} \text {, then }
\end{aligned}
$$

$$
\left\{\begin{array}{l}
\frac{\alpha}{\beta} \geq-\frac{c_{1}}{c_{2}} \Rightarrow a_{1}=a_{2}=\beta \\
\frac{\beta}{\alpha}>-\frac{c_{1}}{c_{2}}>\frac{\alpha}{\beta} \Rightarrow a_{1}=\sqrt{-\frac{\alpha \beta c_{2}}{c_{1}}}, \quad a_{2}=\alpha+\beta-\sqrt{-\frac{\alpha \beta c_{1}}{c_{2}}} \\
-\frac{c_{1}}{c_{2}} \geq \frac{\beta}{\alpha} \Rightarrow a_{1}=a_{2}=\alpha .
\end{array}\right.
$$

Proof. We proceed similarly to Proposition 6.3, but now, in the condition (6.7), the function $\vartheta$ does not necessarily satisfy

$$
\int_{\Omega} \vartheta d x=0
$$

This implies that the function $F$ given in Proposition 6.3 satisfies (6.6) with $r=0$, which easily gives the result.

Second problem. Given a diagonal matrix $\Lambda=\operatorname{diag}(\alpha, \beta)$ with $0<\alpha<\beta$, let us now consider the optimization problem (1.1) when $M(\Omega)$ is the $H$-closure of the matrices of the form $R(x) \Lambda R(x)^{t}$, where $R$ is measurable, and $R(x)$ belongs to $\mathcal{O}_{2}$ for a.e. $x \in \Omega$ (observe that to assume $\Lambda$ diagonal is not a restriction). This set $M(\Omega)$ is known (see, e.g., [25], [16]) and agrees with the set of functions $B \in L^{\infty}\left(\Omega, \mathcal{M}_{2}^{s}\right)$ such that for a.e. $x \in \Omega$, the eigenvalues $b_{1}(x)$ and $b_{2}(x)$ of $B(x)$ satisfy $\alpha \leq b_{1}(x)$, $b_{2}(x) \leq \beta, b_{1}(x) b_{2}(x)=\alpha \beta$. For this choice of $M(\Omega)$, we have the following result.

Proposition 6.7. In the assumptions of Theorem 3.2, if $c_{1}$ and $c_{2}$, with $c_{1} \leq c_{2}$, are the eigenvalues of $C$, then there exists an associated basis $\left\{\mu_{1}, \mu_{2}\right\}$ of eigenvectors of $C$ such that a.e. in $\Omega$, we have

$$
A \mu_{i}=a_{i} \mu_{i}, \quad i=1,2,
$$

where a.e. in the set $\check{\Omega}$ defined by (6.3), the functions $a_{1}, a_{2}$ are given by

$$
c_{2}<0 \quad \text { and } \quad \frac{c_{2}}{c_{1}}>\frac{\alpha}{\beta} \Rightarrow\left\{\begin{array}{l}
a_{1}=\sqrt{\alpha \beta} \sqrt{\frac{c_{2}}{c_{1}}} \\
a_{2}=\sqrt{\alpha \beta} \sqrt{\frac{c_{1}}{c_{2}}}
\end{array}\right.
$$


If $c_{1}>0$ and $\frac{c_{2}}{c_{1}} \leq \frac{\beta}{\alpha}$, then there exist three possibilites:

$$
\left\{\begin{array} { l } 
{ a _ { 1 } = \alpha , } \\
{ a _ { 2 } = \beta , }
\end{array} \text { or } \left\{\begin{array} { l } 
{ a _ { 1 } = \beta , } \\
{ a _ { 2 } = \alpha , }
\end{array} \text { or } \left\{\begin{array}{l}
a_{1}=\sqrt{\alpha \beta} \sqrt{\frac{c_{2}}{c_{1}}}, \\
a_{2}=\sqrt{\alpha \beta} \sqrt{\frac{c_{1}}{c_{2}}} .
\end{array}\right.\right.\right.
$$

In another case $a_{1}=\alpha, a_{2}=\beta$.

Proof. Since the set $M(\Omega)$ is invariable by rotations, we can apply Proposition 5.2 to deduce that for a.e. $x \in \Omega$, there exists a basis $\left\{\mu_{1}(x), \mu_{2}(x)\right\}$ of $\mathbf{R}^{2}$ such that

$$
C(x) \mu_{i}(x)=c_{i}(x) \mu_{i}(x), \quad A(x) \mu_{i}(x)=a_{i}(x) \mu_{i}(x), \quad i=1,2,
$$

with $c_{1}(x) \leq c_{2}(x), a_{1}(x), a_{2}(x) \in \mathbf{R}$. From the definition of $M(\Omega)$, we also have that for a.e. $x \in \Omega$, there exists $t^{*}(x) \in\left[1, \frac{\beta}{\alpha}\right]$ such that

$$
a_{1}(x)=\alpha t^{*}(x), \quad a_{2}(x)=\frac{\beta}{t^{*}(x)} .
$$

Moreover, from (5.1), we deduce that $t^{*}$ satisfies

$$
\begin{aligned}
& \left(t^{*}(x)-t\right)\left(\alpha c_{1}(x)-\frac{\beta c_{2}(x)}{t^{*}(x) t}\right) \\
& \quad+\left(t^{*}(x)-t\right)^{2} \min \left\{\frac{\alpha c_{1}(x)}{t}, \frac{\beta c_{2}(x)}{\left(t^{*}(x)\right)^{2} t}, \frac{1}{t^{*}(x)+t}\left(\alpha c_{1}(x)+\frac{\beta c_{2}(x)}{t^{*}(x) t}\right)\right\} \geq 0
\end{aligned}
$$

for every $t \in\left[1, \frac{\beta}{\alpha}\right]$ and a.e. $x \in \Omega$. In the set where $t^{*}(x)=1$, we have $t^{*}(x)-t<0$ for every $t \in\left(1, \frac{\beta}{\alpha}\right]$. So, dividing by $1-t$ and taking $t$ converging to 1 on the right, we deduce

$$
\alpha c_{1}-\beta c_{2} \leq 0 \quad \text { a.e. in }\left\{x \in \Omega: t^{*}(x)=1\right\} .
$$

Analogously, we deduce

$$
\begin{gathered}
\beta c_{1}-\alpha c_{2} \geq 0 \quad \text { a.e. in }\left\{x \in \Omega: t^{*}(x)=\frac{\beta}{\alpha}\right\}, \\
\alpha c_{1}-\frac{\beta c_{2}}{\left(t^{*}(x)\right)^{2}}=0 \quad \text { a.e. in }\left\{x \in \Omega: 1<t^{*}(x)<\frac{\beta}{\alpha}\right\},
\end{gathered}
$$

where the statement (6.12) implies

$$
c_{1} c_{2}>0, \quad t^{*}=\sqrt{\frac{\beta c_{2}}{\alpha c_{1}}} \text { a.e. in }\left\{x \in \check{\Omega}: 1<t^{*}(x)<\frac{\beta}{\alpha}\right\} .
$$

Analyzing the different cases which appear depending on the sign of $c_{1}$ or $c_{2}$, we easily conclude from (6.10), (6.11), and (6.13) the proof of Proposition 6.7.

Remark 6.8. We have deduced (6.10), (6.11), and (6.13) from inequality (6.9). One could conjecture that this inequality gives, in fact, more information. However, a simple calculus shows that the statements (6.10), (6.11), and (6.13) also imply (6.9).

Remark 6.9. Proposition 6.7 does not give the expressions of $a_{1}$ and $a_{2}$ in the set where $c_{1}>0$ and $\frac{c_{2}}{c_{1}} \leq \frac{\beta}{\alpha}$; it gives three possibilities. The possibility $a_{1}=\alpha, a_{2}=\beta$ 
seems to be the most natural in order to stick continuously with the values of $a_{1}$ and $a_{2}$ in the other zones. We also note that if $M(\Omega)$ was convex then, using that $B-A$ is an admissible direction for every $B \in M(\Omega)$, we should obtain in place of (6.9) that

$$
\alpha t^{*}+\frac{\beta}{t^{*}}=\max \left\{\alpha t+\frac{\beta}{t}: t \in\left[1, \frac{\beta}{\alpha}\right]\right\}
$$

which implies that $t^{*}=1$ (and then $a_{1}=\alpha, a_{2}=\beta$ ) a.e. on the set where $c_{1}>0$ and $\frac{c_{2}}{c_{1}} \leq \frac{\beta}{\alpha}$ as well as the expressions of $a_{1}$ and $a_{2}$ given in Proposition 6.7 in the other cases. However, since $M(\Omega)$ is not convex, this reasoning is not good and thus the only conclusion we obtain is that stated in Proposition 6.7.

\section{REFERENCES}

[1] V. M. Alekseev, V. M. Tikhomirov, and S. V. Fomin, Optimal Control, Consultants Bureau, New York, 1987.

[2] G. Allaire, Homogenization and two-scale convergence, SIAM J. Math. Anal., 23 (1992), pp. $1482-1518$.

[3] G. Allaire, Shape Optimization by the Homogenization Method, Appl. Math. Sci. 146, Springer-Verlag, New York, 2002.

[4] H. Aтtouch, Variational Convergence for Functions and Operators, Applicable Mathematics Series, Pitman, London, 1984.

[5] A. Bensoussan, J. L. Lions, and G. Papanicolau, Asymptotic Analysis for Periodic Structures, Stud. Math. Appl. 5, North-Holland, Amsterdam, 1978.

[6] J. Casado Díaz, J. Couce Calvo, and J. D. Martín Gómez, Sobre el control de la matriz de difusión, in Actas encuentro de matemáticos andaluces (Sevilla, 13-17 November 2000), E. Briales-Morales, A. Carriazo-Rubio, T. Chacón-Rebollo, P. Real-Jurado, and A. RomeroJiménez, eds., Publicaciones de la Universidad de Sevilla, 2001, pp. 237-244.

[7] J. Casado Díaz, J. Couce Calvo, and J. D. Martín Gómez, Sobre la identificación de la matriz de difusión mediante varios experimentos, in Actas XVII CEDYA, VII CMA (Salamanca, 24-28 September 2001), L. Ferragut and A. Santos, eds., 2001, CD-ROM.

[8] A. V. Cherkaev, Variational Methods for Structural Optimization, Appl. Math. Sci. 140, Springer-Verlag, New York, 2000.

[9] J. Deny and J. L. Lions, Les espaces de Beppo Levi, Ann. Inst. Fourier (Grenoble), 5 (19531954), pp. 305-370.

[10] G. Francfort and G. W. Milton, Sets of conductivity and elasticity tensors stable under lamination, Comm. Pure Appl. Math., 47 (1994), pp. 257-279.

[11] A. S. Lewis, Convex analysis on the Hermitian matrices, SIAM J. Optim., 6 (1996), pp. $164-177$.

[12] A. S. Lewis and M. L. Overton, Eigenvalue optimization, in Acta Numerica, Acta Numer. 5, Cambridge University Press, Cambridge, UK, 1996, pp. 149-190.

[13] K. A. LuRIE, Applied Optimal Control Theory of Distributed Systems, Plenum Press, New York, 1993.

[14] K. A. Lurie And A. V. Cherkaev, Exact estimates of the conductivity of a binary mixture of isotropic materials, Proc. Roy. Soc. Edinburgh Sect. A, 104 (1986), pp. 21-38.

[15] G. W. Milton, A link between sets of tensors stable under lamination and quasiconvexity, Comm. Pure Appl. Math., 47 (1994), pp. 959-1003.

[16] G. W. Milton, The Theory of Composites, Cambridge Monogr. Appl. Comput. Math., Cambridge University Press, Cambridge, UK, 2002.

[17] F. Murat, Théorèmes de non-existence pour des problèmes de contrôle dans le coefficients, C. R. Acad. Sci. Paris Sér. A-B, 274 (1972), pp. 395-398.

[18] F. Murat and L. Tartar, On the control of coefficients in partial differential equations, in Topics in the Mathematical Modelling of Composite Materials, Progr. Nonlinear Differential Equations Appl. 31, A. Cherkaev and R. Kohn, eds., Birkhäuser Boston, Boston, 1997, pp. 1-8.

[19] F. Murat and L. Tartar, $H$-convergence, in Topics in the Mathematical Modelling of Composite Materials, Progr. Nonlinear Differential Equations Appl. 31, A. Cherkaev and R. Kohn, eds., Birkhäuser Boston, Boston, 1997, pp. 21-43. 
[20] F. Murat and L. Tartar, Calculus of variations and homogenization, in Topics in the Mathematical Modelling of Composite Materials, Progr. Nonlinear Differential Equations Appl. 31, A. Cherkaev and R. Kohn, eds., Birkhäuser Boston, Boston, 1997, pp. 139-173.

[21] U. Raitums, On the local representation of G-closure, Arch. Ration. Mech. Anal., 158 (2001), pp. 213-234.

[22] S. Spagnolo, Sulla convergenza di soluzioni di equazione paraboliche ed elliptiche, Ann. Scuola Norm. Sup. Pisa (3), 22 (1968), pp. 571-597.

[23] L. TARTAR, Estimations fines de coefficients homogénéisés, in Ennio de Giorgi colloquium (Paris, 1983), Res. Notes Math. 125, P. Kree, ed., Pitman, London, 1985, pp. 168-187.

[24] L. TARTAR, Remarks on optimal design problems, in Calculus of Variations, Homogenization and Continuum Mechanics, Ser. Adv. Math. Appl. Sci. 18, G. Bouchitté, G. Buttazzo, and P. Suquet, eds., World Scientific, Singapore, 1994, pp. 279-296.

[25] L. TARTAR, An Introduction to the homogenization method in optimal design, in Optimal Shape Design (CIM/CIME, Summer School, Trôia, 1-6 June 1998), Lecture Notes in Math. 1740, A. Cellina and A. Ornelas, eds., Springer-Verlag, Berlin, 2000, pp. 47-156. 